\title{
Coexistence and segregation for strongly competing species in special domains
}

\author{
MONICA CONTI ${ }^{\dagger}$ \\ Dipartimento di Matematica "F. Brioschi”, Politecnico di Milano, \\ Via Bonardi 9, I-20133 Milano, Italy \\ VERONICA FELLI ${ }^{\ddagger}$ \\ Dipartimento di Matematica e Applicazioni, Università degli Studi di Milano-Bicocca, \\ Via Cozzi 53, I-20125 Milano, Italy
}

[Received 11 October 2005 and in revised form 19 November 2007]

\begin{abstract}
We deal with strongly competing multispecies systems of Lotka-Volterra type with homogeneous Dirichlet boundary conditions. For a class of nonconvex domains composed of balls connected by thin corridors, we show the occurrence of pattern formation (coexistence and spatial segregation of all the species) as the competition grows indefinitely. As a result we prove the existence and uniqueness of solutions for a remarkable system of differential inequalities involved in segregation phenomena and optimal partition problems.
\end{abstract}

\section{Introduction}

In this paper we consider the system of $k \geqslant 2$ elliptic equations

$$
-\Delta u_{i}=f_{i}\left(x, u_{i}\right)-\kappa u_{i} \sum_{j \neq i} u_{j} \quad \text { in } \Omega
$$

for $i=1, \ldots, k$, where $\Omega \subset \mathbb{R}^{N}$ is a smooth, connected, bounded domain. Systems of this form model the steady states of $k$ organisms which coexist in the area $\Omega$. The function $u_{i}$ represents the population density of the $i$-th species (hence only $u_{i} \geqslant 0$ are considered) and $f_{i}$ describes the internal dynamics of $u_{i}$. The coupling between different equations is the classical Lotka-Volterra interaction term: the positive constant $\kappa$ prescribes the competitive character of the relationship between $u_{i}$ and $u_{j}$ and its largeness measures the strength of the competition.

Systems of this form have attracted considerable attention both in ecology and social science since they furnish a relatively simple model to study phenomena of extinction, coexistence and segregation of states of populations. Several theoretical studies have been carried out in this direction, mainly in the case of two competing species and for the logistic nonlinearities $f_{i}(u)=$ $u\left(a_{i}-u\right)$. We cite for instance [15, 18, 20, 21, 24, 25], where it is shown that both coexistence and exclusion may occur, depending on the relations between the diffusion rates and the coefficients of intraspecific and interspecific competitions.

In this paper we look at the multispecies Lotka-Volterra system (1) from the different perspective investigated in $[5,9,12,13]$ and we study the possibility of coexistence governed by

†E-mail: monica.conti@mate.polimi.it

${ }^{\ddagger}$ E-mail: veronica.felli@unimib.it 
very strong competition. As we shall discuss in detail in Section 3 , the presence of large interactions of competitive type produces the spatial segregation of the densities in the limit configuration as $\kappa \rightarrow \infty$, namely if $\left(u_{i}^{\kappa}\right)_{i=1}^{k}$ solves $\left[1\right.$, then, for all $i=1, \ldots, k, u_{i}^{\kappa}$ converges to some $u_{i}$ in $H^{1}(\Omega)$ which satisfies

$$
u_{i}(x) \cdot u_{j}(x)=0 \quad \text { a.e. in } \Omega, \quad \text { for all } i \neq j,
$$

so that $\left\{u_{i}>0\right\} \cap\left\{u_{j}>0\right\}=\emptyset$. Furthermore, in the limit, the densities satisfy a system of differential inequalities of the form

$$
\begin{cases}-\Delta u_{i} \leqslant f_{i}\left(x, u_{i}\right) & \text { in } \Omega \\ -\Delta \widehat{u}_{i} \geqslant \widehat{f}_{i}\left(x, \widehat{u}_{i}\right) & \text { in } \Omega\end{cases}
$$

where $\widehat{u}_{i}:=u_{i}-\sum_{j \neq i} u_{j}$ and $\widehat{f_{i}}\left(x, \widehat{u}_{i}\right):=f_{i}\left(x, u_{i}\right)-\sum_{j \neq i} f_{j}\left(x, u_{j}\right)$, in the sense of Definition 1.1 below. The link between the differential inequalities $(3)$ and population dynamics is reinforced by considering another class of segregation states between species, governed by a minimization principle rather than strong competition-diffusion. In [3] (see also [2, 4]), the following energy functional was considered:

$$
J(U)=\sum_{i=1}^{k}\left\{\int_{\Omega}\left(\frac{1}{2}\left|\nabla u_{i}(x)\right|^{2}-F_{i}\left(x, u_{i}(x)\right)\right) \mathrm{d} x\right\},
$$

given by the sum of the internal energies of $k$ positive densities $u_{i}$ with internal potentials $F_{i}(x, s)=\int_{0}^{s} f_{i}(x, u) \mathrm{d} u$. The problem of finding the minimum of $J(U)$ in the class of $k$-tuples $U=\left(u_{1}, \ldots, u_{n}\right)$ satisfying $u_{j} \cdot u_{i}=0$ a.e. on $\Omega$ for $i \neq j$ was investigated in [3], where it is proved that any nontrivial minimizer $U$ (if it exists) satisfies the differential inequalities (3).

This further motivates the study of the solutions of (2)-(3) as a natural step in the understanding of segregation phenomena occurring in population dynamics. Remarkably enough, (3) coupled with (2) can be naturally interpreted as a free boundary problem with multiple phases: the unknown free boundary set is given by

$$
\mathcal{F}=\bigcup_{i=1}^{k} \partial\left\{x \in \Omega: u_{i}(x)>0\right\},
$$

which represents the collection of the boundaries of the disjoint supports of the densities. On its support each density $u_{i}$ solves the elliptic equation $-\Delta u_{i}=f_{i}\left(x, u_{i}\right)$, while the free boundary conditions are implicitly contained in the global differential inequalities (3). The study of the properties of $\mathcal{F}$ is important from the ecological point of view since it provides information about how the segregation occurs, in particular about the way the territory is partitioned by the segregated populations. In this direction, in [1- -3, 5, 7] a number of qualitative properties both of $u_{i}$ and the free boundary set $\mathcal{F}$ are exhibited. We refer the interested reader to [6] for a brief review of the regularity theory so far developed, and to the above cited papers for proofs and details.

Another question of particular interest is the existence of a strictly positive solution to (2)-(3), that is, a solution of the differential inequalities 3 with each component $u_{i} \geqslant 0$ and $u_{i}$ positive on a set of positive measure. As a matter of fact, since all the asymptotic states of the Lotka-Volterra system have to satisfy (2)-(3), the existence of such a solution is necessary to ensure that all the species survive under strong competition. It has to be stressed that in [3, 5], the strict positivity is guaranteed by assuming positive boundary values for each component, in the form $u_{i}=\phi_{i}$ on $\partial \Omega$ with $\phi_{i}>0$ on a set of positive $(N-1)$-measure. 
Hence a major problem consists in proving the existence of a positive solution under natural boundary conditions, such as Dirichlet or Neumann homogeneous boundary conditions. This is precisely the problem we face in this paper: we consider (2)- $(3)$ with the Dirichlet condition

$$
u_{i}=0 \quad \text { on } \partial \Omega,
$$

and we look for a strictly positive solution $U=\left(u_{1}, \ldots, u_{k}\right)$. The interesting case of the Neumann condition will be treated elsewhere (see the concluding remarks).

This is an interesting and mathematically challenging problem: we cannot expect in general to avoid extinction of one or more species. For instance, if $\Omega$ is convex, it is shown in [19] that two competing species cannot coexist under strong competition. On the other hand, the main variational procedure leading to solutions of (3), that is, the minimization of the internal energy $J$, may fail under Dirichlet homogeneous conditions, since it in general provides a $k$-tuple of the form $\left(0, \ldots, u_{i}, \ldots, 0\right)$ with all but one component identically zero (see [26] for a similar result). Therefore, some mechanism of different nature must occur in order to ensure coexistence of the species.

In the present paper we show that the geometry of $\Omega$ can play a crucial role in segregation phenomena. In line with [14, 24], where two populations in planar domains of dumbbell shape are dealt with, we consider a class of nonconvex domains $\Omega^{n}, n \in \mathbb{N}$, essentially composed of $k$ balls connected by thin corridors, as depicted in Figure 1 (see [8] and Section 1.1 for the precise definition). Under the main assumption that the Dirichlet problems on each ball admit a

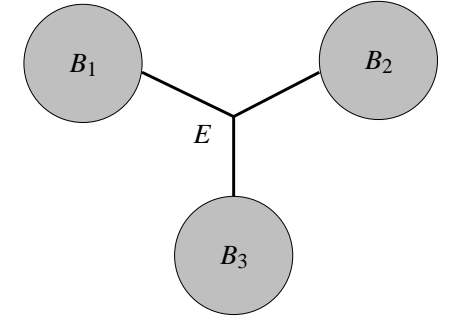

(a) The set $\Omega^{0}=B_{1} \cup B_{2} \cup B_{3}$ and segments $E$ joining the balls.

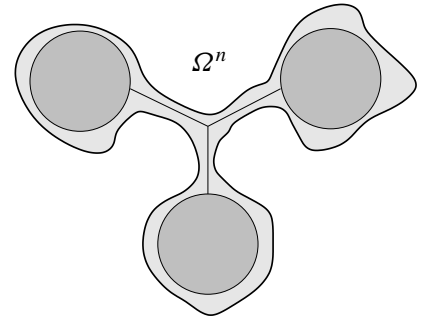

(b) Sets $\Omega$ obtained by small perturbation of $\Omega^{0}$

FIG. 1

nondegenerate local minimizer, we are able to prove existence and uniqueness of positive solutions to the free boundary problem (2)-(3), where each component is close to such a local minimizer (Theorem 1). In ecological terms this means that if in the unperturbed domain and in absence of interaction each species lives in a stable configuration, then strong competition leads to coexistence and segregation of the populations.

Our second result (Theorem 2) concerns the multispecies Lotka-Volterra system endowed with the Dirichlet null condition. Under the same topological and nondegeneracy assumptions, we first prove the existence of a positive solution, provided that the competition parameter $\kappa$ is large enough. This is obtained by exploiting a degree technique introduced by Dancer [8] to control domain perturbation in the case of certain nonlinear equations. Theorem 2 is by itself an interesting result in the framework of multispecies systems. In fact, in contrast to the rich literature dealing with the case 
$k=2$ of two populations, the case of $k \geqslant 3$ species is less understood. We cite for instance [15, 20, 22, 23] for three-species competing systems with cross-diffusion and [10, 11] for the Lotka-Volterra model, where various sufficient conditions for coexistence are provided, depending on the values of the parameters involved.

Next we perform the asymptotic analysis as $\kappa$ grows to infinity, and we prove that this solution converges to the unique segregation state found in Theorem 1. The biological implication of this result is now clear: all the species survive under strong competition in a segregating configuration. Furthermore, as we shall see, they divide the domain in such a way that the $i$-th species does not invade the native territory $B_{j}$ of the other populations. We call this phenomenon the noninvading property.

Both results come from the study of a multispecies system that can be seen as a generalized Lotka-Volterra model with presence of spatial barriers localized in the balls. We shall introduce it in (6), after some rigorous definitions and precise statements of our results.

\subsection{Assumptions and main results}

Let $\Omega^{0}:=\bigcup_{i=1}^{k} B_{i}$ be a finite union of open balls $B_{i} \subset \mathbb{R}^{N}$ such that the $\bar{B}_{i}$ are mutually disjoint, $i=1, \ldots, k$. Following [8], we consider a sequence of domains $\left\{\Omega^{n}\right\}_{n \in \mathbb{N}}$ approximating $\Omega^{0}$ in the following sense: there exists a compact zero measure set $E \subset \mathbb{R}^{N}$ such that

(i) for any compact set $K \subset \Omega^{0}, \Omega^{n} \supset K$ provided $n$ is large;

(ii) for any open set $U \supset E \cup \overline{\Omega^{0}}, \Omega^{n} \subset U$ provided $n$ is large

(see Figure 11. Let us fix a bounded smooth domain $\Omega$ strictly containing $\overline{\Omega^{0}} \cup \overline{\Omega^{n}}$ for all $n \in \mathbb{N}$. Notice that if $\tilde{\Omega} \subset \Omega$ and $u \in H_{0}^{1}(\tilde{\Omega})$, we can extend $u$ to an element of $H_{0}^{1}(\Omega)$ by defining it to be zero outside of $\tilde{\Omega}$. Thus in all the paper we shall think of all our functions as being in $H_{0}^{1}(\Omega)$. We will make the following set of assumptions (for every $i=1, \ldots, k$ ):

(F1) $f_{i}(x, s): \Omega \times \mathbb{R} \rightarrow \mathbb{R}$ is a Carathéodory function, it is odd and $C^{1}$ in the variable $s$, uniformly in $x$;

(F2) $\left|f_{i}^{\prime}(x, s)\right|=O\left(|s|^{q-1}\right)$ for large $|s|$, uniformly in $x$ for some $q<(N+2) /(N-2)(q<\infty$ if $N=2$ ),

where $f_{i}^{\prime}(x, s)=\partial_{s} f_{i}(x, s)$. Furthermore, for any $i=1, \ldots, k$, we assume that the problem

$$
\begin{cases}-\Delta u=f_{i}(x, u) & \text { in } B_{i}, \\ u=0 & \text { on } \partial B_{i},\end{cases}
$$

admits a positive solution $u_{i}^{0} \in H_{0}^{1}\left(B_{i}\right) \cap L^{\infty}\left(B_{i}\right)$ which is nondegenerate in the following sense:

(ND) there exists $\varepsilon>0$ such that

$$
\int_{B_{i}}\left(|\nabla w|^{2}-f_{i}^{\prime}\left(x, u_{i}^{0}\right) w^{2}\right) \mathrm{d} x \geqslant \varepsilon \int_{B_{i}}|\nabla w|^{2} \mathrm{~d} x
$$

for all $w \in H_{0}^{1}\left(B_{i}\right)$ and $i$. 
Note that this implies that the linearized problem at $u_{i}^{0}$,

$$
-\Delta v-f_{i}^{\prime}\left(x, u_{i}^{0}\right) v=0, \quad v \in H_{0}^{1}\left(B_{i}\right),
$$

has only the trivial solution. This is precisely the assumption used in [8]. Condition (ND) is stronger and essentially means that $u_{i}^{0}$ is a local nondegenerate minimizer of the energy on $B_{i}$ (see also [3]). As a model for $f_{i}$ we can consider logistic type nonlinearities $f_{i}(x, s)=\lambda\left(s-|s|^{p-1} s\right), p>1$. It is well known that if $\lambda>\lambda_{1}\left(B_{i}\right)$ (where $\lambda_{1}\left(B_{i}\right)$ is the first eigenvalue of $-\Delta$ in $B_{i}$ with homogeneous Dirichlet boundary conditions) the elliptic problem (5) has a unique positive solution which is a nondegenerate global minimizer for the energy.

Since $u_{i}^{0}$ is an isolated solution to $[5$, the parameter $\delta>0$ will be assumed throughout to be small enough that, for all $i$ :

$$
\text { if } u_{i} \in H_{0}^{1}\left(B_{i}\right) \text { is a solution to (5) such that }\left\|u_{i}-u_{i}^{0}\right\|_{H_{0}^{1}\left(B_{i}\right)} \leqslant \delta \text {, then } u_{i} \equiv u_{i}^{0} \text {. }
$$

We shall also denote by $U^{0}$ the $k$-tuple $\left(u_{1}^{0}, \ldots, u_{k}^{0}\right)$ and by $U=\left(u_{1}, \ldots, u_{k}\right)$ generic $k$-tuples in $\left(H_{0}^{1}\left(\Omega^{n}\right)\right)^{k}$. Let us clarify the meaning of solution to differential inequalities $(3)$ in the following definition.

Definition 1.1 A solution to (3) is a $k$-tuple $U=\left(u_{1}, \ldots, u_{k}\right)$ such that, for every $i=1, \ldots, k$ and $\phi \in H_{0}^{1}(\Omega)$ with $\phi \geqslant 0$ a.e. in $\Omega$,

$$
\int_{\Omega} \nabla u_{i}(x) \cdot \nabla \phi(x) \mathrm{d} x \leqslant \int_{\Omega} f_{i}\left(x, u_{i}(x)\right) \phi(x) \mathrm{d} x
$$

and

$$
\int_{\Omega} \nabla \widehat{u}_{i}(x) \cdot \nabla \phi(x) \mathrm{d} x \geqslant \int_{\Omega} \widehat{f_{i}}\left(x, \widehat{u}_{i}(x)\right) \phi(x) \mathrm{d} x .
$$

Our main theorem ensures the existence of a unique segregated solution to $(3)$ in the perturbed domain $\Omega^{n}$ which is $H^{1}$-close to $U^{0}$.

THEOREM 1 Define

$$
\begin{aligned}
\mathcal{S}\left(\Omega^{n}\right)=\left\{\left(u_{1}, \ldots, u_{k}\right) \in(\right. & \left.H_{0}^{1}\left(\Omega^{n}\right)\right)^{k}: u_{i} \geqslant 0, u_{i} \cdot u_{j}=0 \text { if } i \neq j, \\
& \left.-\Delta u_{i} \leqslant f_{i}\left(x, u_{i}\right),-\Delta \widehat{u}_{i} \geqslant \widehat{f_{i}}\left(x, \widehat{u}_{i}\right), \text { in } \Omega^{n}, i=1, \ldots, k\right\} .
\end{aligned}
$$

Then there exists $\delta>0$ such that, for any $n$ sufficiently large, the class $\mathcal{S}\left(\Omega^{n}\right)$ contains an element $U=\left(u_{1}, \ldots, u_{k}\right) \in\left(H_{0}^{1}\left(\Omega^{n}\right)\right)^{k}$ such that $\left\|u_{i}-u_{i}^{0}\right\|_{H_{0}^{1}\left(\Omega^{n}\right)}<\delta$. Moreover, $u_{i} \equiv 0$ in $B_{j}$ for all $j \neq i$ and $U$ is the unique element of $\mathcal{S}\left(\Omega^{n}\right)$ such that $\left\|U-U^{0}\right\|_{\left(H_{0}^{1}\left(\Omega^{n}\right)\right)^{k}}<\delta$.

As already announced, the proof of Theorem 1 relies on a careful analysis of the following auxiliary system:

$$
\begin{cases}-\Delta u_{i}=f_{i}\left(x, u_{i}\right)-\kappa u_{i} \sum_{j \neq i} u_{j}-\kappa u_{i} \sum_{j \neq i} u_{j}^{0}-\kappa u_{i}^{0} \sum_{j \neq i} u_{j} & \text { in } \Omega^{n}, \\ u_{i}=0 & \text { on } \partial \Omega^{n},\end{cases}
$$

for $i=1, \ldots, k$. 
This system can be seen as a modification of the Lotka-Volterra model, through linear terms which are localized in a single ball each: this feature will be crucial in order to obtain solutions with the noninvading property. Notice that, due to the presence of the barriers, systems (6) fail to satisfy the maximum principle, so that we cannot ensure the positivity of solutions nor even, by now, the competitive character of the model. As we shall see, this will cause some technical difficulties.

Nonetheless, by careful energy estimates and eigenvalue theory, the system for fixed $\kappa$ will be shown to be suitably nondegenerate on $\Omega^{n}$ if $n$ is large enough. This will allow us the application of the degree technique introduced in $[8]$ to control domain perturbation, and as a result we will obtain the existence of a solution $U^{\kappa}$ of the system, which is close to $U^{0}$. The major feature of this approach is that the whole procedure turns out to be uniform with respect to $\kappa$. This uniformity will allow us to perform successfully the asymptotic analysis of the solutions to the auxiliary system as the competition parameter goes to infinity. The final result can be collected in the following form.

THEOREM 2 There exists $\delta>0$ such that, for any $\kappa$ and $n$ sufficiently large, both the LotkaVolterra system (1) with Dirichlet boundary conditions (4) on $\Omega^{n}$ and the modified model (6) admit a solution $U^{\kappa}=\left(u_{1}^{\kappa}, \ldots, u_{k}^{\kappa}\right) \in\left(H_{0}^{1}\left(\Omega^{n}\right)\right)^{k}$ such that $\left\|U^{\kappa}-U^{0}\right\|_{\left(H_{0}^{1}\left(\Omega^{n}\right)\right)^{k}}<\delta$, and, in the case of (1), $U^{\kappa}$ is strictly positive. Furthermore, as $\kappa \rightarrow \infty, u_{i}^{\kappa} \rightarrow u_{i}$ strongly in $H^{1}\left(\Omega^{n}\right)$, where the $k$-tuple $U=\left(u_{1}, \ldots, u_{k}\right)$ is the unique element in $\mathcal{S}\left(\Omega^{n}\right)$ close to $U^{0}$.

\subsection{Plan of the paper}

In Section 2 we establish some preliminary facts to be used throughout the paper, in particular we discuss the nondegeneracy of the problems in $\Omega^{0}$. Section 3 is devoted to the asymptotic analysis of the solutions to the auxiliary system as $\kappa \rightarrow \infty$. In Section 4 we prove the uniqueness of the solution to (3) close to $U^{0}$, as stated in the uniqueness part of Theorem 1 . Section 5 is devoted to the proof of the existence of a solution $U$ close to $U^{0}$ for system [6, when the domain is close enough to $\Omega^{0}$ and the competition is large. We conclude Section 5 by presenting the proofs of Theorems 1 and 2 and giving some final remarks. A final appendix collects some technical proofs and lemmas used throughout the paper.

\section{Preliminary results}

In this section we further modify the Lotka-Volterra system in order to ensure sign conditions and boundedness of its solutions. Furthermore we derive from condition (ND) the main nondegeneracy properties holding in the unperturbed set $\Omega^{0}$.

Let us consider the following system:

$$
\begin{cases}-\Delta u_{i}=f_{i}\left(x,\left[u_{i}+u_{i}^{0}\right]^{+}-u_{i}^{0}\right)-\kappa\left[u_{i}+u_{i}^{0}\right]^{+} \sum_{j \neq i}\left[u_{j}+u_{j}^{0}\right]^{+} & \text {in } \tilde{\Omega}, \\ u_{i}=0 & \text { on } \partial \tilde{\Omega},\end{cases}
$$

for $i=1, \ldots, k$, where $\Omega^{0} \subset \tilde{\Omega} \subseteq \Omega$. Here and throughout, the symbol $[t(x)]^{+}$will denote the positive part of $t$, that is, $[t(x)]^{+}=\max \{t(x), 0\}$. The motivation for this choice is contained in the following lemma.

Lemma 2.1 Let $U=\left(u_{1}, \ldots, u_{k}\right)$ be a solution of system (7). Then $u_{i} \geqslant-u_{i}^{0}$ in $\tilde{\Omega}$ for all $i=1, \ldots, k$. In particular the $u_{i}$ solve (6) and satisfy $u_{i}(x) \geqslant 0$ for $x \in B_{j}$ when $j \neq i$. 
Proof. Let $v_{i}=u_{i}+u_{i}^{0}$. It satisfies the equation

$$
-\Delta v_{i}=f_{i}\left(x,\left[u_{i}+u_{i}^{0}\right]^{+}-u_{i}^{0}\right)+f_{i}\left(x, u_{i}^{0}\right)-\kappa\left[v_{i}\right]^{+} \sum_{j \neq i}\left[v_{j}\right]^{+} .
$$

Now it suffices to test this equation with $-\left[u_{i}+u_{i}^{0}\right]^{-}$, recalling that $f_{i}$ is odd.

REMARK 2.1 Notice that the original system (1) can be recovered in this model by the formal identification $u_{i}^{0} \equiv 0$. In particular, by Lemma 2.1 it turns out that the solutions obtained with $f_{i}\left(x,\left[u_{i}\right]^{+}\right)$instead of $f_{i}\left(x, u_{i}\right)$ satisfy $u_{i} \geqslant 0$ for all $i$, and thus are nonnegative solutions for the Lotka-Volterra system (1).

\subsection{Differential inequalities}

Let $\left(u_{1}, \ldots, u_{k}\right)$ be a solution to (7). Since by Lemma 2.1 each $u_{i}$ satisfies $u_{i}+u_{i}^{0} \geqslant 0$, the coupling term has negative sign and we immediately have

$$
-\Delta u_{i} \leqslant f_{i}\left(x, u_{i}\right) .
$$

Furthermore, by a straightforward calculation we obtain an opposite differential inequality for $\widehat{u}_{i}$ :

$$
-\Delta \widehat{u}_{i} \geqslant f_{i}\left(x, u_{i}\right)-\sum_{j \neq i} f_{j}\left(x, u_{j}\right)=\widehat{f_{i}}\left(x, \widehat{u}_{i}\right) .
$$

Thus the solutions of (7) satisfy the differential inequalities 3 .

\subsection{Uniform $L^{\infty}$ bounds}

We now suitably modify $f_{i}$ in order to ensure that the solutions of the new system are bounded in $L^{\infty}$. This is based on the following result due to Dancer [8]: for $n$ sufficiently large, the problem

$$
\begin{cases}-\Delta u=f_{i}(x, u) & \text { in } \Omega^{n}, \\ u=0 & \text { on } \partial \Omega^{n},\end{cases}
$$

admits a positive solution $\phi_{i}^{n} \in H_{0}^{1}\left(\Omega^{n}\right)$ which is close to $\sum u_{i}^{0}$ in some $L^{r}(\Omega)(r>1)$. Fix $n_{0}$ large such that $\Omega^{n} \subset \Omega^{n_{0}}$ for all $n \geqslant n_{0}$ and denote $\phi_{i}^{n_{0}}$ simply by $\phi_{i}$. Define

$$
\tilde{f}_{i}(x, s)= \begin{cases}f_{i}(x, s) & \text { if } s \leqslant \phi_{i}(x), \\ f_{i}\left(x, \phi_{i}(x)\right) & \text { if } s>\phi_{i}(x) .\end{cases}
$$

Lemma 2.2 For $n \geqslant n_{0}$, let $u_{i} \in H_{0}^{1}\left(\Omega^{n}\right)$ be such that $-\Delta u_{i} \leqslant \tilde{f}_{i}\left(x, u_{i}\right)$ in $\Omega^{n}$. Then $u_{i} \leqslant \phi_{i}$ a.e. in $\Omega$.

Proof. Summing up the differential inequalities for $\phi_{i}$ and $u_{i}$ gives

$$
\begin{cases}-\Delta\left(\phi_{i}-u_{i}\right) \geqslant f_{i}\left(x, \phi_{i}\right)-\tilde{f}_{i}\left(x, u_{i}\right) & \text { in } \Omega^{n} \\ \phi_{i}-u_{i} \geqslant 0 & \text { on } \partial \Omega^{n} .\end{cases}
$$


Set $\omega=\left\{x \in \Omega^{n}: \phi_{i}<u_{i}\right\}$; note that $\omega$ is strictly contained in $\Omega^{n}$ by the boundary conditions. Hence by testing the first inequality with $-\left(\phi_{i}-u_{i}\right)^{-}$we obtain

$$
\int_{\omega}\left|\nabla\left(\phi_{i}-u_{i}\right)^{-}\right|^{2} \mathrm{~d} x \leqslant-\int_{\omega}\left(f_{i}\left(x, \phi_{i}\right)-\tilde{f}_{i}\left(x, u_{i}\right)\right)\left(\phi_{i}-u_{i}\right)^{-} \mathrm{d} x=0,
$$

which implies $\left(\phi_{i}-u_{i}\right)^{-}=0$ and so $\phi_{i} \geqslant u_{i}$.

As a consequence, any solution $\left(u_{1}, \ldots, u_{k}\right)$ to either (7) or (3), with $\tilde{f}_{i}$ instead of $f_{i}$ in $\Omega^{n}$, satisfies

$$
-u_{i}^{0} \leqslant u_{i} \leqslant \phi_{i}, \quad i=1, \ldots, k .
$$

In particular, any solution with $u_{i}$ close to $u_{i}^{0}$ will be a true solution of the original problem with $f_{i}$. Moreover, by the classical strong maximum principle and Harnack's inequality, any solution $\left(u_{1}, \ldots, u_{k}\right)$ to (7) satisfies either $u_{i} \equiv \phi_{i}$, or $u_{i} \equiv-u_{i}^{0}$, or $-u_{i}^{0}<u_{i}<\phi_{i}$.

Notations. Throughout the paper we shall work with $\tilde{f}_{i}$ instead of $f_{i}$, denoting $\tilde{f}_{i}$ simply by $f_{i}$; supp $u_{i}$ will denote the set $\left\{u_{i}>0\right\}$.

\subsection{Nondegeneracy in $\Omega^{0}$}

Let us now consider (7) in $\Omega^{0}$. Then we immediately realize that $U^{0}=\left(u_{1}^{0}, \ldots, u_{k}^{0}\right)$ is a solution of the problem. Furthermore, it follows from (ND) that $U^{0}$ is an isolated solution, uniformly in $\kappa$.

THEOREM 2.1 There exist $\bar{\kappa}>0$ and $\delta>0$ such that if $U^{\kappa}$ is a solution of (7) in $\Omega^{0}$ such that $\left\|U^{\kappa}-U^{0}\right\|<\delta$ in $\left(H_{0}^{1}\left(\Omega^{0}\right)\right)^{k}$, then $U^{\kappa} \equiv U^{0}$ for all $\kappa \geqslant \bar{\kappa}$.

An analogous result holds for the solutions to (2)-(3), thanks to the following sign condition prescribed by the validity of 9 .

LEMMA 2.3 Let $\left(u_{1}, \ldots, u_{k}\right)$ be a solution of $-\Delta \widehat{u}_{i} \geqslant \widehat{f}\left(x, \widehat{u}_{i}\right)$ in $B_{i}$ for some $i$. Assume that $u_{i} \cdot u_{j}=0$ if $i \neq j$ and that $\left\|u_{j}-u_{j}^{0}\right\|_{H_{0}^{1}(\Omega)} \leqslant \delta$ for all $j=1, \ldots, k$. Then, if $\delta$ is small enough, $\widehat{u}_{i} \geqslant 0$.

THEOREM 2.2 Let $\left(u_{1}, \ldots, u_{k}\right) \in \mathcal{S}\left(\Omega^{0}\right)$ be such that $\left\|u_{i}-u_{i}^{0}\right\|_{H_{0}^{1}\left(\Omega^{0}\right)} \leqslant \delta$ for all $i=1, \ldots, k$. Then, if $\delta$ is small enough, $u_{i} \equiv u_{i}^{0}$ for all $i=1, \ldots, k$.

All these results are crucial in what follows, but since the proofs are somewhat technical, we postpone them to the Appendix.

\section{Asymptotic analysis as $\kappa \rightarrow \infty$}

This section is devoted to establishing the link between population systems and the original set of differential inequalities (3). To this end, throughout the section let $\delta>0$ and assume that there exists a solution $\left(u_{1}^{\kappa}, \ldots, u_{k}^{\kappa}\right)$ to (7) such that $\left\|u_{i}^{\kappa}-u_{i}^{0}\right\|_{H^{1}(\Omega)} \leqslant \delta$ for all large $\kappa$. Our main result is

THEOREM 3.1 Let $\tilde{\Omega}$ be a connected domain such that $\Omega^{0} \subset \tilde{\Omega} \subseteq \Omega$. For each $\kappa$ let $U^{\kappa}=$ $\left(u_{1}^{\kappa}, \ldots, u_{k}^{\kappa}\right)$ be a solution of 77 in $\tilde{\Omega}$ such that $\left\|U^{\kappa}-U^{0}\right\|_{\left(H_{0}^{1}(\Omega)\right)^{k}}<\bar{\delta}$. Then, if $\delta$ is small enough, there exists $U \in\left(H_{0}^{1}(\tilde{\Omega})\right)^{k}$ such that, for all $i=1, \ldots, k$ : 
(i) up to a subsequence, $u_{i}^{\kappa} \rightarrow u_{i}$ strongly in $H^{1}(\Omega)$ as $\kappa \rightarrow \infty$,

(ii) $u_{i}>0$ in $B_{i}$,

(iii) if $i \neq j$ then $u_{i}=0$ a.e. in $B_{j}$,

(iv) if $i \neq j$ then $u_{i} \cdot u_{j}=0$ a.e. in $\Omega$,

(v) $\left(u_{1}, \ldots, u_{k}\right)$ satisfy the differential inequalities $(3)$.

The proof of this fact is obtained through the next Lemmas 3.1 3.3 .

LEMma 3.1 Under the same assumptions of Theorem 3.1 if $\delta$ is small enough there exists $U \in$ $\left(H_{0}^{1}(\tilde{\Omega})\right)^{k}$ such that, for all $i=1, \ldots, k$ :

(i) up to a subsequence, $u_{i}^{\kappa} \rightarrow u_{i}$ weakly in $H^{1}(\Omega)$ as $\kappa \rightarrow \infty$,

(ii) $u_{i} \geqslant 0$ in $\Omega \backslash B_{i}$,

(iii) if $i \neq j$ then $u_{i}=0$ a.e. in $B_{j}$,

(iv) if $i \neq j$ then $u_{i} \cdot u_{j}=0$ a.e. in $\Omega$,

(v) $\left(u_{1}, \ldots, u_{k}\right)$ satisfy the differential inequalities $(3)$.

Proof. Since $U^{\kappa}$ is bounded in $\left(H^{1}(\Omega)\right)^{k}$ by assumption, we immediately obtain the existence of a weak limit $U$ such that, up to subsequences, $u_{i}^{\kappa} \rightarrow u_{i}$ in $H^{1}(\Omega)$. Since each $u_{i}^{\kappa}$ is positive on $B_{j}$ when $j \neq i$ by Lemma 2.1. property (ii) comes from almost everywhere pointwise convergence. Furthermore, the differential inequalities (8) and (9) for $u_{i}^{\kappa}$ pass to the weak limit, so (v) is already proved. Let us discuss properties (iii) and (iv). By testing $[6]$ with $u_{i}^{\kappa}+u_{i}^{0}$ we see that

$$
\kappa \int_{\Omega}\left(u_{i}^{\kappa}+u_{i}^{0}\right)^{2} \sum_{j \neq i}\left(u_{j}^{\kappa}+u_{j}^{0}\right) \quad \text { is bounded uniformly in } \kappa,
$$

and hence, since $u_{j}^{\kappa}+u_{j}^{0} \geqslant 0$ for all $j$,

$$
\int_{\Omega}\left(u_{i}^{\kappa}+u_{i}^{0}\right)^{2} \sum_{j \neq i}\left(u_{j}^{\kappa}+u_{j}^{0}\right) \rightarrow 0 \quad \text { as } \kappa \rightarrow \infty .
$$

Passing to the limit for $U^{\kappa} \rightarrow U$ we obtain, for all $i \neq j, i, j=1, \ldots, k$,

$$
u_{i}(x) \cdot u_{j}(x)+u_{i}^{0}(x) \cdot u_{j}(x)+u_{i}(x) \cdot u_{j}^{0}(x)=0, \quad \forall x \in \Omega .
$$

Let $x \in \tilde{\Omega} \backslash \bigcup B_{i}$. Then (11) ensures $u_{i}(x) \cdot u_{j}(x)=0$ for all $i \neq j$.

Claim. If $x \in B_{i}$ then $u_{j}(x)=0$ for all $j \neq i$.

Let $x \in B_{i}$ for some fixed $i$. If $u_{i}(x)=0$ then 111 becomes $u_{i}^{0}(x) \cdot u_{j}(x)=0$ and hence $u_{j}(x)=0$ for all $j \neq i$. If $u_{i}(x)>-u_{i}^{0}(x)$ we have $u_{j}(x)\left(u_{i}(x)+u_{i}^{0}(x)\right)=0$, implying again $u_{j}(x)=0$ for all $j \neq i$. Finally, let $u_{i}(x)=-u_{i}^{0}(x)$. Since $u_{j} \cdot u_{h}=0$ in $B_{i}$ for all $j \neq h, j, h \neq i$, there exists at most one index different from $i$ (say $j$ ) where $u_{j}(x)>0$. Let $\omega_{j}$ be the connected component of $\left\{u_{j}>0\right\}$ which is contained in the set $\left\{y \in B_{i}: u_{i}(y)=-u_{i}^{0}(y)\right\}$, and such that $x \in \omega_{j}$. Then by 9 , since $u_{h}=0$ in $\omega_{j}$ for all $h \neq i, j$, we have

$$
-\Delta\left(u_{i}-u_{j}\right) \geqslant f_{i}\left(\cdot, u_{i}\right)-f_{j}\left(\cdot, u_{j}\right) \quad \text { in } \omega_{j} .
$$


Adding $-\Delta u_{i}^{0}=f_{i}\left(\cdot, u_{i}^{0}\right)$ we get

$$
-\Delta\left(u_{i}-u_{j}+u_{i}^{0}\right) \geqslant f_{i}\left(\cdot, u_{i}\right)-f_{j}\left(\cdot, u_{j}\right)+f_{i}\left(\cdot, u_{i}^{0}\right) \quad \text { in } \omega_{j} .
$$

Test this equation with $-\left[u_{i}-u_{j}+u_{i}^{0}\right]^{-}$. Note that $\left.\left[u_{i}-u_{j}+u_{i}^{0}\right]^{-} \equiv u_{j}\right|_{\omega_{j}}$, so

$$
\int_{\omega_{j}}\left|\nabla u_{j}\right|^{2} \leqslant \int_{\omega_{j}} f_{j}\left(\cdot, u_{j}\right) u_{j} \leqslant\left\|f_{j}\left(\cdot, u_{j}\right)\right\|_{L^{N / 2}\left(\omega_{j}\right)}\left\|u_{j}\right\|_{L^{2^{*}\left(\omega_{j}\right)}}^{2}
$$

By (F1), (F2), since $\left\|u_{j}\right\|_{H^{1}\left(\omega_{j}\right)} \leqslant \delta$, we have $\left\|f_{j}\left(\cdot, u_{j}\right)\right\|_{L^{N / 2}\left(\omega_{j}\right)} \leqslant C \delta$, which implies $u_{j} \equiv 0$ in $\omega_{j}$ if $\delta$ is small enough, giving rise to a contradiction. This proves the claim.

LEMMA 3.2 Under the assumptions of Theorem 3.1, if $\delta$ is small enough, then $u_{i}>0$ in the whole of $B_{i}$. In particular, $B_{i} \subset \operatorname{supp} u_{i}$.

Proof. By Theorem 3.1, we already know that $u_{i} \geqslant 0$ in $\Omega \backslash B_{i}$. Furthermore, $u_{i} \cdot u_{j}=0$ for $i \neq j$ in $\Omega$ and $\left(u_{1}, \ldots, u_{k}\right)$ satisfies $[9]$. Therefore Lemma 2.3 yields $\widehat{u}_{i}=u_{i}-\sum_{j \neq i} u_{j} \geqslant 0$ in $B_{i}$. Since by Theorem 3.1 (ii), $u_{j} \geqslant 0$ in $B_{i}$, we have $u_{i} \geqslant 0$ in $B_{i}$. Hence $u_{i} \geqslant 0$ in $\Omega$ and it is not identically null by its closeness to $u_{i}^{0}$; the strict positivity now comes from the Harnack inequality.

LEMMA 3.3 Under the assumptions of Theorem 3.1 the convergence $u_{i}^{\kappa} \rightarrow u_{i}$ is strong in $H_{0}^{1}(\Omega)$ (up to subsequences), where $U=\left(u_{1}, \ldots, u_{k}\right)$ is as in Lemma 3.1

Proof. In order to prove the strong convergence of $u_{i}^{\kappa}$ to $u_{i}$ in $H_{0}^{1}(\Omega)$, consider the functions $\hat{u}_{i}=u_{i}-\sum_{j \neq i} u_{j}$, which satisfy the inequality (9) in $\tilde{\Omega}$. Since $u_{i} \geqslant 0$ from Lemma 3.2 , testing (9) with $u_{i}$ we obtain

$$
\int_{\Omega} u_{i} f_{i}\left(\cdot, u_{i}\right) \leqslant \int_{\Omega}\left|\nabla u_{i}\right|^{2}
$$

Testing

$$
-\Delta u_{i}^{\kappa} \leqslant f_{i}\left(x, u_{i}^{\kappa}\right) \quad \text { in } \tilde{\Omega}
$$

with $u_{i}^{\kappa}+u_{i}^{0}$ (which is positive in view of Lemma 2.1) we have

$$
\int_{\Omega} \nabla u_{i}^{\kappa} \cdot \nabla u_{i}^{0}+\int_{\Omega}\left|\nabla u_{i}^{\kappa}\right|^{2} \leqslant \int_{\Omega} u_{i}^{0} f_{i}\left(\cdot, u_{i}^{\kappa}\right)+\int_{\Omega} u_{i}^{\kappa} f_{i}\left(\cdot, u_{i}^{\kappa}\right) .
$$

The uniform $L^{\infty}$-bound provided in Section 2.2 and the dominated convergence theorem allow us to pass to the limit in 13 to obtain

$$
\int_{B_{i}} \nabla u_{i}^{0} \cdot \nabla u_{i}+\limsup _{\kappa \rightarrow \infty} \int_{\Omega}\left|\nabla u_{i}^{\kappa}\right|^{2} \leqslant \int_{B_{i}} u_{i}^{0} f_{i}\left(\cdot, u_{i}\right)+\int_{\Omega} u_{i} f_{i}\left(\cdot, u_{i}\right) .
$$

Since by Theorem 3.1, $u_{i}$ solves $-\Delta u_{i}=f_{i}\left(x, u_{i}\right)$ in $B_{i}$, testing with $u_{i}^{0}$ we have $\int_{B_{i}} \nabla u_{i} \cdot \nabla u_{i}^{0}=$ $\int_{B_{i}} u_{i}^{0} f_{i}\left(\cdot, u_{i}\right)$, which implies

$$
\limsup _{\kappa \rightarrow \infty} \int_{\Omega}\left|\nabla u_{i}^{\kappa}\right|^{2} \leqslant \int_{\Omega} u_{i} f_{i}\left(\cdot, u_{i}\right)
$$

Now (12), 14), and the lower semicontinuity of the norms yield

$$
\lim _{\kappa \rightarrow \infty} \int_{\Omega}\left|\nabla u_{i}^{\kappa}\right|^{2}=\int_{\Omega}\left|\nabla u_{i}\right|^{2} .
$$

The strong convergence follows easily from weak convergence and convergence of norms. 
REMARK 3.1 Notice that the above analysis can also be performed for the Lotka-Volterra system (1), with some differences. In particular, following the proof of Lemma 3.1, the segregation property (iv) immediately follows from (11), which reduces in this case to $u_{i} \cdot u_{j}=0$. In contrast, we cannot prove, at the moment, the noninvading property (iii).

\section{Uniqueness of the asymptotic limit}

As in the previous section, let us here assume that the system (7) does have a solution on $\Omega^{n}$ for all $\kappa$ large. Our goal now is to prove that the class $\mathcal{S}\left(\Omega^{n}\right)$ contains a single element which is close to $U^{0}$; it is worth noticing that $U^{0}$ does not belong to $\mathcal{S}\left(\Omega^{n}\right)$, since the differential inequalities involving the hat operation cannot hold outside $\Omega^{0}$.

THEOREM 4.1 For $\delta$ sufficiently small and $n$ sufficiently large, the class $\mathcal{S}\left(\Omega^{n}\right)$ has at most one element $U$ such that $\left\|U-U^{0}\right\|_{\left(H_{0}^{1}(\Omega)\right)^{k}}<\delta$.

Proof. By Theorem 3.1, let $U^{n} \in \mathcal{S}\left(\Omega^{n}\right)$ be the asymptotic limit of the solutions to 77 , so that $U^{n}$ enjoys the noninvading property. Now assume by contradiction the existence of $V^{n} \in \mathcal{S}\left(\Omega^{n}\right)$ such that $U^{n} \neq V^{n}$.

Claim 1 As $n \rightarrow \infty$, both $U^{n} \rightarrow U^{0}$ and $V^{n} \rightarrow U^{0}$ weakly in $H_{0}^{1}(\Omega)$ (hence strongly in $L^{p}(\Omega)$ for all $1 \leqslant p<2^{*}$ ).

It suffices to prove the claim for $U^{n}$. Since $U^{n}$ is bounded in $\left(H^{1}(\Omega)\right)^{k}$, there exists $U \in$ $\left(H^{1}(\Omega)\right)^{k}$ such that $u_{i}^{n} \rightarrow u_{i}$ weakly in $H^{1}(\Omega)$, and strongly in all $L^{p}(\Omega)$ with subcritical $p$. We are going to prove that $U \in \mathcal{S}\left(\Omega^{0}\right)$ so that $U \equiv U^{0}$ in light of Lemma 2.2 To this end, notice that the differential inequalities characterizing $\mathcal{S}\left(\Omega^{0}\right)$ are satisfied by $u_{i}^{n}$ for all $n$, hence they pass to the weak limit. It remains to prove that $u_{i} \in H_{0}^{1}\left(\Omega^{0}\right)$. To see this, notice that, for all open sets $\mathcal{V}$ containing $\overline{\Omega^{0}} \cup E$, we have

$$
\operatorname{supp} u_{i}^{n} \subset \Omega^{n} \subset \mathcal{V},
$$

provided that $n$ is sufficiently large. Hence

$$
\operatorname{supp} u_{i} \subset \mathcal{V} \quad \text { for all open sets } \mathcal{V} \text { containing } \overline{\Omega^{0}} \cup E,
$$

which implies that $u_{i}=0$ a.e. in $\Omega \backslash\left(\overline{\Omega^{0}} \cup E\right)$. Since $\partial \Omega \cup E$ has measure zero, $u_{i}=0$ on $\Omega \backslash \overline{\Omega^{0}}$, and the smoothness of $\partial \Omega^{0}$ ensures that $u_{i} \in H_{0}^{1}\left(\Omega^{0}\right)$ (see [17]).

Let us now start the argument that will lead to a contradiction. By setting $\omega_{i}^{n}=\left\{u_{i}^{n}>0\right\}$, we have $\widehat{u}_{i}^{n}=u_{i}^{n}$ in $\omega_{i}^{n}$ and

$$
\begin{array}{ll}
-\Delta u_{i}^{n}=f_{i}\left(x, u_{i}^{n}\right) & \text { in } \omega_{i}^{n}, \\
-\Delta v_{i}^{n} \leqslant f_{i}\left(x, v_{i}^{n}\right) & \text { in } \omega_{i}^{n} .
\end{array}
$$

If we now consider

we have

$$
w_{i}^{n}=\frac{v_{i}^{n}-u_{i}^{n}}{\left\|V^{n}-U^{n}\right\|_{L^{2}(\Omega)}},
$$

$$
\begin{cases}-\Delta w_{i}^{n} \leqslant a_{i}^{n}(x) w_{i}^{n} & \text { in } \omega_{i}^{n} \\ -\Delta \widehat{w}_{i}^{n} \geqslant b_{i}^{n}(x) \widehat{w}_{i}^{n} & \text { in } \omega_{i}^{n}\end{cases}
$$


where

$$
a_{i}^{n}(x)=\frac{f_{i}\left(x, v_{i}^{n}\right)-f_{i}\left(x, u_{i}^{n}\right)}{v_{i}^{n}-u_{i}^{n}} \quad \text { and } \quad b_{i}^{n}(x)=\frac{\widehat{f_{i}}\left(x, \widehat{v}_{i}^{n}\right)-f_{i}\left(x, u_{i}^{n}\right)}{\widehat{v}_{i}^{n}-u_{i}^{n}} \text {. }
$$

Notice that $a_{i}^{n} \in L^{\infty}$ independently of $n$ in light of the a priori estimates in 10 and Lemma 2.2 and since $f_{i}^{\prime}(\cdot, 0)$ is bounded. We assert that this is also true for the second quotient. To see this, remember that $v_{i}^{n} \cdot v_{j}^{n}=0$ in $\Omega$ and notice that

$$
b_{i}^{n}(x)=\frac{f_{i}\left(x, v_{i}^{n}(x)\right)-f_{i}\left(x, u_{i}^{n}(x)\right)}{v_{i}^{n}(x)-u_{i}^{n}(x)}
$$

for $x \in \Omega$ such that $v_{i}^{n}(x)>0$. On the other hand, if $v_{j}(x)>0$ for some $j \neq i$, then

$$
b_{i}^{n}(x)=\frac{f_{i}\left(x, u_{i}^{n}(x)\right)+f_{j}\left(x, v_{j}^{n}(x)\right)}{u_{i}^{n}(x)+v_{j}^{n}(x)} .
$$

Hence the same argument used to estimate $a_{i}^{n}$ provides an $L^{\infty}$ control for $b_{i}^{n}$, uniformly in $n$. As a consequence, by testing the differential inequalities in $(15)$ with $\left[w_{i}^{n}\right]^{+}$and $-\left[\widehat{w}_{i}^{n}\right]^{-}$respectively, we easily find that $w_{i}^{n}$ is bounded in $H^{1}(\Omega)$. Since this is true for all $i=1, \ldots, k$, there exists $W=\left(w_{1}, \ldots, w_{k}\right) \in\left(H^{1}(\Omega)\right)^{k}$ such that $w_{i}^{n} \rightarrow w_{i}$ weakly in $H^{1}(\Omega)$ and strongly in $L^{2}$ so that $w_{i} \neq 0$ for some $i$.

CLAIM $2 w_{i} \in H_{0}^{1}\left(B_{i}\right)$ and $-\Delta w_{i} \leqslant f_{i}^{\prime}\left(x, u_{i}^{0}\right) w_{i}$ in $B_{i}$.

Reasoning as in Claim 1, we can easily prove that $w_{i} \in H_{0}^{1}\left(B_{i}\right)$.

Let $\phi \geqslant 0$ be such that $\phi \in C_{0}^{\infty}\left(B_{i}\right)$. Since by Theorem 3.2 we know that $B_{i} \subset \omega_{i}^{n}$, we can test the first inequality in (15) with $\phi$ to obtain

$$
\int_{B_{i}}\left[\nabla w_{i}^{n} \nabla \phi-a_{i}^{n}(x) w_{i}^{n} \phi\right] \leqslant 0 .
$$

By the strong convergence of $U^{n}$ and $V^{n}$ to $U^{0}$ in $L^{p}(\Omega)$ for all $1 \leqslant p<2^{*}$ and by the continuity of the Nemytskiu operator $f_{i}^{\prime}: L^{N(q-1) / 2}(\Omega) \rightarrow L^{N / 2}(\Omega)$ (see $(25)$ ), it is easy to realize that $a_{i}^{n} \rightarrow f_{i}^{\prime}\left(\cdot, u_{i}^{0}\right)$ in $L^{N / 2}(\Omega)$ as $n \rightarrow \infty$. Hence we can pass to the limit to find

$$
\int_{B_{i}}\left[\nabla w_{i} \nabla \phi-f_{i}^{\prime}\left(x, u_{i}^{0}\right) w_{i} \phi\right] \leqslant 0 .
$$

By exploiting the same argument starting from the second inequality in 15 , we can prove the opposite inequality, $-\Delta w_{i} \geqslant f_{i}^{\prime}\left(x, u_{i}^{0}\right) w_{i}$ in $B_{i}$. To this end, we first notice that if we set $A_{n}^{i, j}:=\left\{x \in B_{i}: v_{j}^{n}(x)>0\right\}$, then $\lim _{n \rightarrow \infty} \mu\left(A_{n}^{j}\right)=0$ if $j \neq i$.

Indeed, $A_{n}^{i, j} \subset\left\{x \in \Omega \backslash B_{j}: v_{i}^{n}(x)=0\right\}$ for every $j \neq i$, so we have

$$
o(1)=\int_{B_{i}}\left|u_{i}^{0}-v_{i}^{n}\right|^{2} \mathrm{~d} x \geqslant \int_{\left\{x \in B_{i}: v_{i}^{n}(x)=0\right\}}\left|u_{i}^{0}\right|^{2} \mathrm{~d} x \geqslant \int_{A_{n}^{i, j}}\left|u_{i}^{0}\right|^{2} \mathrm{~d} x
$$

as $n \rightarrow \infty$. From the absolute continuity of the Lebesgue measure $\mu$ with respect to the measure $A \mapsto \int_{A}\left|u_{i}^{0}\right|^{2} \mathrm{~d} x$ in $B_{i}$, we deduce that

$$
\lim _{n \rightarrow \infty} \mu\left(A_{n}^{i, j}\right)=0
$$


In $B_{i}$ we can write $b_{i}^{n}$ as

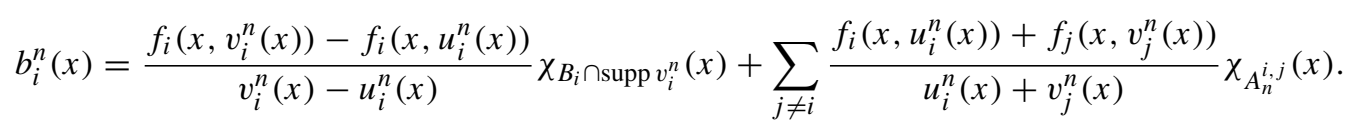

From $\lim _{n \rightarrow \infty} \mu\left(A_{n}^{i, j}\right)=0$, the a priori estimates in (10) and Lemma 2.2. and since $f_{i}^{\prime}(\cdot, 0)$ is bounded, we deduce that $b_{i}^{n}(x) \rightarrow f_{i}^{\prime}\left(x, u_{i}^{0}(x)\right)$ for a.e. $x \in B_{i}$. From the uniform $L^{\infty}$-boundedness of $b_{i}^{n}$ and the dominated convergence theorem, we conclude that $b_{i}^{n} \rightarrow f_{i}^{\prime}\left(\cdot, u_{i}^{0}\right)$ in $L^{N / 2}(\Omega)$ as $n \rightarrow \infty$. Hence testing the second inequality in (15) with $\phi \in C_{0}^{\infty}\left(B_{i}\right), \phi \geqslant 0$, and passing to the limit as $n \rightarrow \infty$ we obtain

$$
\int_{B_{i}}\left[\nabla \widehat{w}_{i} \nabla \phi-f_{i}^{\prime}\left(x, u_{i}^{0}\right) \widehat{w}_{i} \phi\right] \geqslant 0 .
$$

From 17 and $L^{2}$-convergence of $w_{j}^{n}$ to $w_{j}$, for all $j \neq i$ we have

$$
\int_{B_{i}}\left|w_{j}\right|^{2} \mathrm{~d} x=\lim _{n \rightarrow \infty} \int_{B_{i}}\left|w_{j}^{n}\right|^{2} \mathrm{~d} x=\lim _{n \rightarrow \infty} \int_{A_{n}^{i, j}}\left|w_{j}^{n}\right|^{2} \mathrm{~d} x=0 .
$$

Therefore $w_{j}=0$ a.e. in $B_{i}$ for every $j \neq i$, and

$$
\widehat{w}_{i}=w_{i} \quad \text { in } B_{i} .
$$

From (16), (18), and [19), we conclude that $w_{i}$ is a nontrivial solution to the linearized equation $-\Delta w_{i}=f_{i}^{\prime}\left(x, u_{i}^{0}\right) w_{i}$ in $B_{i}$ with boundary condition $w_{i}=0$ on $\partial B_{i}$. This provides a contradiction with the nondegeneracy assumption (ND).

REMARK 4.1 We note that the weak $H^{1}(\Omega)$-convergence stated in Claim 1 of the above proof is actually strong. Indeed, from Theorem 3.1 (v), we have

$$
\left\|u_{i}^{n}\right\|_{H^{1}(\Omega)}^{2}=\int_{\operatorname{supp} u_{i}^{n}}\left|\nabla u_{i}^{n}\right|^{2} \mathrm{~d} x=\int_{\Omega} \chi_{\operatorname{supp} u_{i}^{n}}(x) f_{i}\left(x, u_{i}^{n}(x)\right) u_{i}^{n}(x) \mathrm{d} x .
$$

By the choice of $\Omega^{n}$, Theorem 3.1 (iii), and pointwise convergence of $u_{i}^{n}$ to $u_{i}^{0}$, it follows that $\chi_{\text {supp } u_{i}^{n}} \rightarrow \chi_{B_{i}}$ a.e. in $\Omega$. Hence the uniform $L^{\infty}$-bound provided in Section 2.2 and the dominated convergence theorem allow passing to the limit on the right hand side to obtain

$$
\lim _{n \rightarrow \infty}\left\|u_{i}^{n}\right\|_{H^{1}(\Omega)}^{2}=\int_{B_{i}} f_{i}\left(x, u_{i}^{0}(x)\right) u_{i}^{0}(x) \mathrm{d} x=\left\|u_{i}^{0}\right\|_{H^{1}(\Omega)}^{2} .
$$

Strong $H^{1}(\Omega)$-convergence now follows from weak convergence and convergence of norms.

\section{Coexistence in Lotka-Volterra models}

This section is devoted to proving the existence of solutions to the auxiliary system when the domain is sufficiently close to $\Omega^{0}$ and the interspecific competition is sufficiently strong. Precisely, we shall prove 
THEOREM 5.1 For any $\kappa$ and $n$ sufficiently large, the system with barriers $(6)$ admits a solution $U^{\kappa}=\left(u_{1}^{\kappa}, \ldots, u_{k}^{\kappa}\right) \in\left(H_{0}^{1}\left(\Omega^{n}\right)\right)^{k}$ which is close to $U^{0}=\left(u_{1}^{0}, \ldots, u_{k}^{0}\right)$ in $\left(H_{0}^{1}\left(\Omega^{n}\right)\right)^{k}$.

In light of Remark 2.1 the above theorem immediately yields

COROLlaRY 5.1 For any $\kappa$ and $n$ sufficiently large, the Lotka-Volterra system (1) admits a solution $U^{\kappa}=\left(u_{1}^{\kappa}, \ldots, u_{k}^{\kappa}\right) \in\left(H_{0}^{1}\left(\Omega^{n}\right)\right)^{k}$ which is close to $U^{0}=\left(u_{1}^{0}, \ldots, u_{k}^{0}\right)$ in $\left(H_{0}^{1}\left(\Omega^{n}\right)\right)^{k}$ and satisfies $u_{i} \geqslant 0$ for all $i$.

The proof of Theorem 5.1 is obtained by using a standard topological degree technique (see e.g. [16]) and it is based on the ideas introduced in [8] in order to control the perturbation of the domain. As a first step, we introduce suitable operators which allow reformulating the existence of solutions to (7) as a fixed point problem. For all integers $n=0,1, \ldots$, we define

$$
A^{n, \kappa}:\left(H_{0}^{1}(\Omega)\right)^{k} \rightarrow\left(H_{0}^{1}(\Omega)\right)^{k}, \quad A^{n, \kappa}:=L^{n} \circ F^{n, \kappa} \circ i^{n},
$$

where $i^{n}:\left(H_{0}^{1}(\Omega)\right)^{k} \rightarrow\left(H^{1}\left(\Omega^{n}\right)\right)^{k}$ is the restriction $i^{n}\left(u_{1}, \ldots, u_{k}\right)=\left(u_{1}\left|\Omega^{n}, \ldots, u_{k}\right| \Omega^{n}\right)$,

$$
\begin{aligned}
& F^{n, \kappa}:\left(H^{1}\left(\Omega^{n}\right)\right)^{k} \rightarrow\left(H^{-1}\left(\Omega^{n}\right)\right)^{k}, \\
& F^{n, \kappa}(U)=f_{i}\left(\cdot,\left[u_{i}+u_{i}^{0}\right]^{+}-u_{i}^{0}\right)-\kappa\left[u_{i}+u_{i}^{0}\right]^{+} \sum_{j \neq i}\left[u_{j}+u_{j}^{0}\right]^{+},
\end{aligned}
$$

and

$$
L^{n}:\left(H^{-1}\left(\Omega^{n}\right)\right)^{k} \rightarrow\left(H_{0}^{1}\left(\Omega^{n}\right)\right)^{k} \hookrightarrow\left(H_{0}^{1}(\Omega)\right)^{k}
$$

is defined as: $L^{n}\left(h_{1}, \ldots, h_{k}\right)=\left(u_{1}, \ldots, u_{k}\right)$ if and only if $-\Delta u_{i}=h_{i}$ in $\Omega^{n}$ and $u_{i}=0$ on $\partial \Omega^{n}$, for all $i=1, \ldots, k$.

With the above notation, it turns out that the solutions of (7) in $\Omega^{n}$ are in 1-1 correspondence with the fixed points of $A^{n, \kappa}$. We are going to prove the existence of fixed points of $A^{n, \kappa}$ by showing that the Leray-Schauder degree of the map Id $-A^{n, \kappa}$ in a small ball centered at $U^{0}$ is different from 0 . We recall that the Leray-Schauder degree is well defined for operators which differ from the identity by a compact map. To this end, we notice that it is not restrictive to assume that $A^{n, \kappa}$ is compact from $\left(H_{0}^{1}(\Omega)\right)^{k}$ into itself. Indeed, if $N<6$, the growth of the nonlinearity $\tilde{q}=\max \{2, q\}$ is subcritical, i.e. $\tilde{q}<(N+2) /(N-2)$, and compactness is guaranteed by the Sobolev-Rellich embedding theorem. Otherwise, for $N \geqslant 6$, using the $L^{\infty}$-bounds proved in Section 2.2. compactness can be recovered by truncating the coupling term, thus obtaining a subcritical nonlinearity without affecting the proofs.

The following lemma allows us to compute the topological degree of the unperturbed problem. We will use the notation $A^{\prime}(U)$ to denote the Fréchet derivative at $U \in X$ of any differentiable map $A$ from a Banach space $X$ to a Banach space $Y$.

Lemma 5.1 Let $\varepsilon>0$ be as in assumption (ND). There exists $\bar{\kappa}$ such that for all $\kappa>\bar{\kappa}$, the eigenvalues of Id $-\left(A^{0, \kappa}\right)^{\prime}\left(U^{0}\right)$ in $\left(H_{0}^{1}\left(\Omega^{0}\right)\right)^{k}$ are all greater than $\varepsilon$. In particular, the kernel of $\mathrm{Id}-\left(A^{0, \kappa}\right)^{\prime}\left(U^{0}\right)$ is trivial.

Proof. First notice that, by Lemma 6.2 in the Appendix, the map

$$
F^{0, \kappa}:\left(H^{1}\left(\Omega^{0}\right)\right)^{k} \rightarrow\left(H^{-1}\left(\Omega^{0}\right)\right)^{k}
$$


is Fréchet differentiable at $U^{0}$ and

$$
\left(F^{0, \kappa}\right)^{\prime}\left(U^{0}\right)[V]=\operatorname{Jac} G^{\kappa}\left(U^{0}\right) V \quad \text { for all } V \in\left(H^{1}\left(\Omega^{0}\right)\right)^{k},
$$

where

$$
G^{\kappa}: \mathbb{R}^{k} \rightarrow \mathbb{R}^{k}, \quad G^{\kappa}(U)=f_{i}\left(\cdot, u_{i}\right)-\kappa u_{i} \sum_{j \neq i} u_{j}-\kappa u_{i}^{0} \sum_{j \neq i} u_{j}-\kappa u_{i} \sum_{j \neq i} u_{j}^{0},
$$

and Jac $G^{\kappa}\left(U^{0}\right)$ denotes the Jacobian matrix of $G^{\kappa}$ at $U^{0}$.

Set $\mathcal{L}_{\kappa}:=\operatorname{Id}-\left(A^{0, \kappa}\right)^{\prime}\left(U^{0}\right)$ and write $\left(H_{0}^{1}\left(\Omega^{0}\right)\right)^{k}$ as the direct sum

$$
\left(H_{0}^{1}\left(\Omega^{0}\right)\right)^{k}=\bigoplus_{i=1}^{k} \mathcal{H}_{i}
$$

where

$$
\mathcal{H}_{i}=H_{0}^{1}\left(B_{i}\right) \times H_{0}^{1}\left(B_{i+1}(\bmod k)\right) \times H_{0}^{1}\left(B_{i+2(\bmod k)}\right) \times \cdots \times H_{0}^{1}\left(B_{i+k-1(\bmod k)}\right) .
$$

The spaces $\mathcal{H}_{i}$ are mutually orthogonal and $\left.\mathcal{L}_{\kappa}\right|_{\mathcal{H}_{i}}: \mathcal{H}_{i} \rightarrow \mathcal{H}_{i}$, so that it is enough to prove that 0 is not an eigenvalue of $\mathcal{L}_{\kappa} \mid \mathcal{H}_{i}$ for all $i=1, \ldots, k$.

If $\lambda$ is an eigenvalue of $\mathcal{L}_{\kappa}$ in $\mathcal{H}_{1}$, then there exists $V=\left(v_{1}, \ldots, v_{k}\right) \in \mathcal{H}_{1}$ such that $\left(v_{1}, \ldots, v_{k}\right) \neq(0, \ldots, 0)$ and

$$
-(1-\lambda) \Delta V=\operatorname{Jac} G^{\kappa}\left(U^{0}\right) V,
$$

i.e.

$$
-(1-\lambda) \Delta v_{i}=\left(f_{i}^{\prime}\left(\cdot, u_{i}^{0}\right)-2 \kappa \sum_{j \neq i} u_{j}^{0}\right) v_{i}-2 \kappa u_{i}^{0} \sum_{j \neq i} v_{j} \quad \text { in } \Omega^{0}
$$

for all $i=1, \ldots, k$. Since $\left(v_{1}, \ldots, v_{k}\right) \neq(0, \ldots, 0)$, there exists $\ell$ such that $v_{\ell} \not \equiv 0$. Equation 21] for $i=\ell$ in $B_{\ell}$ reads

$$
-(1-\lambda) \Delta v_{\ell}=f_{\ell}^{\prime}\left(\cdot, u_{\ell}^{0}\right) v_{\ell} \quad \text { in } B_{\ell},
$$

hence $\lambda \geqslant \varepsilon$ in view of assumption (ND).

If $\lambda$ is an eigenvalue of $\mathcal{L}_{\kappa}$ in $\mathcal{H}_{i}$ for $i \neq 1$, then there exists $V=\left(v_{1}, \ldots, v_{k}\right) \in \mathcal{H}_{i}, V \neq$ $(0, \ldots, 0)$, which solves 211 . Let $\ell$ be such that $v_{\ell} \not \equiv 0$. Then equation (21) in $B_{i+\ell-1}$ reads

$$
-(1-\lambda) \Delta v_{\ell}=\left(f_{\ell}^{\prime}(\cdot, 0)-2 \kappa u_{i+\ell-1}^{0}\right) v_{\ell}, \quad v_{\ell} \in H_{0}^{1}\left(B_{i+\ell-1}\right) .
$$

Testing the above equation with $v_{\ell}$ we find

$$
\begin{aligned}
(1-\lambda) \int_{B_{i+\ell-1}}\left|\nabla v_{\ell}\right|^{2} \mathrm{~d} x & =\int_{B_{i+\ell-1}}\left(f_{\ell}^{\prime}(\cdot, 0)-2 \kappa u_{i+\ell-1}^{0}\right) v_{\ell}^{2} \mathrm{~d} x \\
& \leqslant \int_{B_{i+\ell-1}}\left(f_{\ell}^{\prime}(\cdot, 0)-2 \kappa u_{i+\ell-1}^{0}\right)^{+} v_{\ell}^{2} \mathrm{~d} x \\
& \leqslant S^{-1}\left(\int_{B_{i+\ell-1}}\left|\nabla v_{\ell}\right|^{2} \mathrm{~d} x\right)\left\|\left(f_{\ell}^{\prime}(\cdot, 0)-2 \kappa u_{i+\ell-1}^{0}\right)^{+}\right\|_{L^{N / 2}\left(B_{i+\ell-1}\right)}
\end{aligned}
$$


where $S$ is the best constant in the Sobolev embedding. Therefore

$$
\lambda \geqslant 1-S^{-1}\left\|\left(f_{\ell}^{\prime}(\cdot, 0)-2 \kappa u_{i+\ell-1}^{0}\right)^{+}\right\|_{L^{N / 2}\left(B_{i+\ell-1}\right)} .
$$

By the dominated convergence theorem, $\left\|\left(f_{\ell}^{\prime}(\cdot, 0)-\kappa u_{i+\ell-1}^{0}\right)^{+}\right\|_{L^{N / 2}\left(B_{i+\ell-1}\right)} \rightarrow 0$ as $\kappa \rightarrow \infty$ for any $\ell$ and $i$, hence we can find $\bar{\kappa}$ such that for all $\kappa \geqslant \bar{\kappa}$, and all $i$ and $\ell$,

$$
\left\|\left(f_{\ell}^{\prime}(\cdot, 0)-2 \kappa u_{i+\ell-1}^{0}\right)^{+}\right\|_{L^{N / 2}\left(B_{i+\ell-1}\right)}<S(1-\varepsilon) .
$$

With this choice of $\bar{\kappa}$, from $(22)$ it follows that if $\lambda$ is an eigenvalue of $\mathcal{L}_{\kappa}$ in $\mathcal{H}_{i}$ for $i \neq 1$, then $\lambda \geqslant \varepsilon ;$ in particular $\lambda \neq 0$. The proof is thereby complete.

LEMMA 5.2 There exist $\bar{\kappa}$ and $\bar{n}$ such that, for all $n \geqslant \bar{n}$ and $\kappa \geqslant \bar{\kappa}$,

$$
U \neq t A^{0, \kappa}(U)+(1-t) A^{n, \kappa}(U)
$$

for all $t \in[0,1]$ and $U \in\left(H_{0}^{1}(\Omega)\right)^{k}$ such that $\left\|U-U^{0}\right\|_{\left(H_{0}^{1}(\Omega)\right)^{k}}=\delta$.

Proof. Arguing by contradiction, suppose there exist sequences $n_{j} \rightarrow \infty$ and $\kappa_{j} \rightarrow \infty, t_{j} \in[0,1]$, and $U^{j} \in\left(H_{0}^{1}(\Omega)\right)^{k}$ such that $\left\|U^{j}-U^{0}\right\|_{\left(H_{0}^{1}(\Omega)\right)^{k}}=\delta$ and

$$
U^{j}=t_{j} A^{0, \kappa_{j}}\left(U^{j}\right)+\left(1-t_{j}\right) A^{n_{j}, \kappa_{j}}\left(U^{j}\right) .
$$

Since $A^{n_{j}, \kappa_{j}}$ takes values in $\left(H_{0}^{1}\left(\Omega^{n_{j}}\right)\right)^{k}$, we see that $U^{j} \in\left(H_{0}^{1}\left(\Omega^{n_{j}}\right)\right)^{k}$. Taking the laplacian of both sides in 23 , we find that $U^{j}$ solves

$$
\left\{\begin{array}{l}
-\Delta U^{j}=F^{\kappa_{j}}\left(U^{j}\right), \\
U^{j} \in\left(H_{0}^{1}\left(\Omega^{n_{j}}\right)\right)^{k} .
\end{array}\right.
$$

Since $\left\{U^{j}\right\}_{j}$ is bounded in $\left(H_{0}^{1}(\Omega)\right)^{k}$, up to a subsequence, $U^{j}$ converges weakly in $\left(H_{0}^{1}(\Omega)\right)^{k}$ to some $U=\left(u_{1}, \ldots, u_{k}\right) \in\left(H_{0}^{1}(\Omega)\right)^{k}$. By Theorem 3.1, we know that $u_{i} \cdot u_{j}=0$ for $i \neq j, u_{i} \geqslant 0$ in $\Omega$ and the $k$-tuple $\left(u_{1}, \ldots, u_{k}\right)$ solves the differential inequality $(9)$, i.e.

$$
-\Delta \widehat{u}_{i} \geqslant \widehat{f}_{i}\left(x, \widehat{u}_{i}\right) \quad \text { in } \Omega \text {. }
$$

As a matter of fact, arguing as in Theorem 4.1 (see the proof of Claim 1), it is possible to prove that $u_{i} \in H_{0}^{1}\left(\Omega^{0}\right)$, hence $U \in \mathcal{S}\left(\Omega^{0}\right)$. Furthermore, since the convergence of $U^{j}$ to $U$ is actually strong in $\left(H_{0}^{1}(\Omega)\right)^{k}$ by Lemma 3.3 , we have $\sum_{i}\left\|u_{i}-u_{i}^{0}\right\|_{H_{0}^{1}\left(B_{i}\right)}^{2}=\left\|U-U^{0}\right\|_{\left(H_{0}^{1}(\Omega)\right)^{k}}^{2}=\delta^{2}>0$. This implies the existence of $i$ such that $u_{i} \not \equiv u_{i}^{0}$, in contradiction with Theorem 2.2

We now have all the ingredients to conclude the proof of Theorem 5.1

Proof of Theorem 5.1. In view of Theorem 2.1 for all $\kappa \geqslant \bar{\kappa}$ we can compute the Leray-Schauder degree

$$
\operatorname{deg}\left(\operatorname{Id}-A^{0, \kappa}, B_{\left(H_{0}^{1}(\Omega)\right)^{k}}\left(U^{0}, \delta\right), 0\right) .
$$

By Lemma 5.1 it turns out to be equal to +1 . In light of Lemma 5.2, for $n \geqslant \bar{n}$ and $\kappa \geqslant \bar{\kappa}$, it makes sense to compute the Leray-Schauder degree

$$
I=\operatorname{deg}\left(\operatorname{Id}-A^{n, \kappa}, B_{\left(H_{0}^{1}(\Omega)\right)^{k}}\left(U^{0}, \delta\right), 0\right) .
$$


By homotopy invariance,

$$
\operatorname{deg}\left(\operatorname{Id}-A^{n, \kappa}, B_{\left(H_{0}^{1}(\Omega)\right)^{k}}\left(U^{0}, \delta\right), 0\right)=\operatorname{deg}\left(\operatorname{Id}-A^{0, \kappa}, B_{\left(H_{0}^{1}(\Omega)\right)^{k}}\left(U^{0}, \delta\right), 0\right)
$$

and hence $I=+1$. As a consequence, $A^{n, \kappa}$ has a fixed point in $B_{\left(H_{0}^{1}(\Omega)\right)^{k}}\left(U^{0}, \delta\right)$, which provides a solution $U=\left(u_{1}, \ldots, u_{k}\right)$ to 77 in $\Omega^{n}$ which is close to $U^{0}$. To conclude the proof, it only remains to show that $U$ is a solution to $(6)$ with the original nonlinearity $f_{i}$, and this follows easily from (10).

Collecting all the results so far obtained, we can finally prove our main theorems.

Proof of Theorem 1. For a fixed sufficiently large $n$, consider the sequence $U^{\kappa}$ of solutions to (6) as in Theorem 5.1 As $\kappa \rightarrow \infty$, thanks to Theorem 3.1 we know that $U^{\kappa}$ converges strongly to some $U=\left(u_{1}, \ldots, u_{n}\right)$ in $\left(H_{0}^{1}\left(\Omega^{n}\right)\right)^{k}$ such that $U$ is $H^{1}$-close to $U^{0}, u_{i} \geqslant 0$ for all $i, u_{i} \cdot u_{j}=0$ if $i \neq j$, $U$ has the noninvading property and satisfies the differential inequalities $(3$. Hence $U$ belongs to $\mathcal{S}\left(\Omega^{n}\right)$. The uniqueness is ensured by Theorem 4.1 .

Proof of Theorem 2. The existence of a solution $U^{\kappa}$ close to $U^{0}$ for the two systems is proved in Theorem 5.1 and the subsequent corollary. The asymptotic analysis as $\kappa \rightarrow \infty$ has been carried out for (6) in Section 3 and all the results directly come from Theorem 3.1. For the Lotka-Volterra model (1), $U^{k}$ converges to an element of $\mathcal{S}\left(\Omega^{n}\right)$ by Remark 3.1

\section{$5.1 \quad$ Concluding remarks}

In this paper we have restricted our discussion to homogeneous Dirichlet boundary conditions. It has to be stressed that the technique here employed cannot be used to treat the Neumann no-flux boundary conditions

$$
\frac{\partial u_{i}}{\partial v}=0 \quad \text { on } \partial \Omega^{n}
$$

the two major obstacles being the difficulty in constructing suitable extension operators and the lack of continuity of the eigenvalues of the Laplacian under Neumann boundary conditions with respect to the perturbation of the domain. This will be an object of forthcoming studies.

On the other hand, our results can be immediately extended to a great variety of competitive models, not necessarily of Lotka-Volterra type, since they essentially depend only on the validity of the differential inequalities 9 .

\section{Appendix}

In this appendix, we collect some lemmas used throughout the paper and the proofs of our most technical results. The following simple lemma is needed to prove Theorem 2.1 i.e. to prove isolation of $U^{0}$.

LEMMA 6.1 For all $\varepsilon>0$ there exists $\delta>0$ such that for all $i=1, \ldots, k$ and $u \in H_{0}^{1}\left(\Omega^{0}\right)$, $\left\|u-u_{i}^{0}\right\|_{H_{0}^{1}\left(\Omega^{0}\right)} \leqslant \delta$ implies

$$
\left|\int_{\Omega^{0}}\left(f_{i}\left(x, u_{i}\right)-f_{i}\left(x, u_{i}^{0}\right)-f_{i}^{\prime}\left(x, u_{i}^{0}\right)\left(u_{i}-u_{i}^{0}\right)\right)\left(u_{i}-u_{i}^{0}\right) \mathrm{d} x\right| \leqslant \varepsilon\left\|u_{i}-u_{i}^{0}\right\|_{H_{0}^{1}\left(\Omega^{0}\right)}^{2} .
$$


Proof. Denote by $I$ the integral in (24). We can estimate it as follows:

$$
\begin{aligned}
I & =\left|\int_{\Omega^{0}}\left[\int_{0}^{1}\left(f_{i}^{\prime}\left(x, t u_{i}+(1-t) u_{i}^{0}\right)-f_{i}^{\prime}\left(x, u_{i}^{0}\right)\right)\left(u_{i}-u_{i}^{0}\right)^{2} \mathrm{~d} t\right] \mathrm{d} x\right| \\
& \leqslant\left\|u_{i}-u_{i}^{0}\right\|_{L^{2^{*}}\left(\Omega^{0}\right)}^{2} \int_{0}^{1}\left\|f_{i}^{\prime}\left(\cdot, t u_{i}+(1-t) u_{i}^{0}\right)-f_{i}^{\prime}\left(\cdot, u_{i}^{0}\right)\right\|_{L^{N / 2}\left(\Omega^{0}\right)} \mathrm{d} t \\
& \leqslant S^{-1}\left\|u_{i}-u_{i}^{0}\right\|_{H_{0}^{1}\left(\Omega^{0}\right)}^{2} \int_{0}^{1}\left\|f_{i}^{\prime}\left(\cdot, t u_{i}+(1-t) u_{i}^{0}\right)-f_{i}^{\prime}\left(\cdot, u_{i}^{0}\right)\right\|_{L^{N / 2}\left(\Omega^{0}\right)} \mathrm{d} t,
\end{aligned}
$$

where $S$ is the best constant of the Sobolev embedding $H_{0}^{1} \hookrightarrow L^{2^{*}}$. By continuity of the Nemytskiu operator $f_{i}^{\prime}: H_{0}^{1}\left(\Omega^{0}\right) \rightarrow L^{N / 2}\left(\Omega^{0}\right), u \mapsto f_{i}^{\prime}(\cdot, u(\cdot))$, there exists $\delta>0$ such that

$$
\left\|w-u_{0}^{i}\right\|_{H_{0}^{1}\left(\Omega^{0}\right)} \leqslant \delta \Rightarrow\left\|f_{i}^{\prime}(\cdot, w)-f_{i}^{\prime}\left(\cdot, u_{i}^{0}\right)\right\|_{L^{N / 2}\left(\Omega^{0}\right)} \leqslant S \varepsilon .
$$

This completes the proof.

Let us now prove Theorem 2.1, which has played a crucial role in the degree argument developed in Section 5, as it ensures the isolation of the solution to the unperturbed problem.

Proof of Theorem 2.1. Assume that there exists a sequence $U^{\kappa}=\left(u_{1}^{\kappa}, \ldots, u_{k}^{\kappa}\right)$ of solutions to (6) such that $u_{i}^{\kappa}>-u_{i}^{0}$ for all $i$ and $U^{\kappa} \rightarrow U^{0}$ as $\kappa \rightarrow \infty$. Set $V^{\kappa}=U^{\kappa}-U^{0}$. By subtracting the respective differential equations we obtain, for all $i=1, \ldots, k$,

$$
-\Delta v_{i}^{\kappa}=f_{i}\left(x, u_{i}^{\kappa}\right)-f_{i}\left(x, u_{i}^{0}\right)-\kappa v_{i}^{\kappa} \sum_{j \neq i} v_{j}^{\kappa}-2 \kappa u_{i}^{\kappa} \sum_{j \neq i} u_{j}^{0}-2 \kappa u_{i}^{0} \sum_{j \neq i} u_{j}^{\kappa} \quad \text { in } \Omega^{0} .
$$

Add and subtract the term $f_{i}^{\prime}\left(x, u_{i}^{0}\right) v_{i}^{\kappa}$, then multiply by $v_{i}^{\kappa}$ and integrate on $B_{h}$ for a fixed $h$ to obtain

$$
\begin{aligned}
\int_{B_{h}}\left[\left|\nabla v_{i}^{\kappa}\right|^{2}-f_{i}^{\prime}\left(x, u_{i}^{0}\right)\left|v_{i}^{\kappa}\right|^{2}-\left(f_{i}\left(x, u_{i}^{\kappa}\right)-f_{i}\left(x, u_{i}^{0}\right)-f_{i}^{\prime}\left(x, u_{i}^{0}\right) v_{i}^{\kappa}\right) v_{i}^{\kappa}\right. \\
\left.+2 \kappa u_{i}^{0}\left(\sum_{j \neq i} v_{j}^{\kappa}\right) v_{i}^{\kappa}+2 \kappa\left(\sum_{j \neq i} u_{j}^{0}\right)\left|v_{i}^{\kappa}\right|^{2}+\kappa\left(\sum_{j \neq i} v_{j}^{\kappa}\right)\left|v_{i}^{\kappa}\right|^{2}\right]=0 .
\end{aligned}
$$

In particular, since $\left.v_{j}\right|_{B_{h}}=u_{j}$ if $j \neq h$ while $\left.v_{h}\right|_{B_{h}}=u_{h}-u_{h}^{0}$, by choosing $h \neq i$ we have

$$
\int_{B_{h}}\left|\nabla v_{i}^{\kappa}\right|^{2}-\int_{B_{h}}\left(f_{i}\left(x, u_{i}^{\kappa}\right)-f_{i}(x, 0)-f_{i}^{\prime}(x, 0) v_{i}^{\kappa}\right) v_{i}^{\kappa}=\int_{B_{h}}\left(f_{i}^{\prime}(x, 0)-\kappa u_{h}^{0}-\kappa \sum_{j \neq i} u_{j}^{\kappa}\right)\left|v_{i}^{\kappa}\right|^{2} .
$$

Let $0<\varepsilon<1$ be given. If $\kappa$ is large enough, in light of Lemma 6.1 and since $u_{i}(x)>0$ for $x \in B_{j}$ when $j \neq i$, we know that

$$
\begin{aligned}
(1-\varepsilon) \int_{B_{h}}\left|\nabla v_{i}^{\kappa}\right|^{2} & \leqslant \int_{B_{h}}\left[f_{i}^{\prime}(x, 0)-\kappa\left(u_{h}^{\kappa}+u_{h}^{0}\right)\right]^{+}\left|v_{i}^{\kappa}\right|^{2} \\
& \leqslant\left\|\left[f_{i}^{\prime}(x, 0)-\kappa\left(u_{h}^{\kappa}+u_{h}^{0}\right)\right]^{+}\right\|_{L^{N / 2}\left(B_{h}\right)}\left\|v_{i}^{\kappa}\right\|_{L^{2^{*}\left(B_{h}\right)}}^{2}
\end{aligned}
$$


Claim. The $L^{N / 2}$-norm of $\left[f_{i}^{\prime}(\cdot, 0)-\kappa\left(u_{h}^{\kappa}+u_{h}^{0}\right)\right]^{+}$can be made arbitrarily small by letting $\kappa \rightarrow \infty$.

First note that $u_{h}^{\kappa}+u_{h}^{0}>0$ by assumption, hence

$$
\left\|\left[f_{i}^{\prime}(\cdot, 0)-\kappa\left(u_{h}^{\kappa}+u_{h}^{0}\right)\right]^{+}\right\|_{L^{N / 2}(\omega)}<\left\|\left[f_{i}^{\prime}(\cdot, 0)\right]^{+}\right\|_{L^{N / 2}(\omega)} \leqslant\left(\sup _{x \in \Omega^{0}}\left[f_{i}^{\prime}(x, 0)\right]^{+}\right) \mu(\omega)^{2 / N}
$$

for any measurable $\omega \subset \Omega^{0}$. Secondly, since $\left\|u_{h}^{\kappa}-u_{h}^{0}\right\|_{H_{0}^{1}\left(\Omega^{0}\right)} \leqslant \delta$, by the Sobolev embedding we have

$$
\delta^{2} \geqslant \int_{\Omega^{0}}\left|\nabla\left(u_{h}^{\kappa}-u_{h}^{0}\right)\right|^{2} \geqslant S\left(\int_{A_{\delta}}\left|u_{h}^{\kappa}-u_{h}^{0}\right|^{2^{*}}\right)^{2 / 2^{*}} \geqslant \delta \mu\left(A_{\delta}^{\kappa}\right)^{2 / 2^{*}}
$$

where

$$
A_{\delta}^{\kappa}=\left\{x \in B_{h}:\left|u_{h}^{\kappa}(x)-u_{h}^{0}(x)\right|^{2}>\delta\right\} .
$$

Choose $\delta$ (independent of $\kappa$ ) small enough so that

$$
\left(\sup _{x \in \Omega^{0}}\left[f_{i}^{\prime}(x, 0)\right]^{+}\right)^{N / 2} \cdot \mu\left(A_{\delta}^{\kappa}\right) \leqslant \frac{1}{4}(S(1-\varepsilon))^{N / 2} .
$$

Now fix $r>0$ such that

$$
\left(\sup _{x \in \Omega^{0}}\left[f_{i}^{\prime}(x, 0)\right]^{+}\right)^{N / 2} \cdot \mu\left(B_{h} \backslash B_{h}(r)\right) \leqslant \frac{1}{4}(S(1-\varepsilon))^{N / 2},
$$

where $B_{h}(r)$ denotes the ball of radius $r$ and with the same center as $B_{h}$. We note that there exists $m>0$ such that $u_{h}^{0}(x) \geqslant m$ for all $x \in B_{h}(r)$. Also, for $0<\sqrt{\delta}<m / 2$, we have $u_{h}^{\kappa}+u_{h}^{0}>m / 2$ in $B_{h}(r) \backslash A_{\delta}^{\kappa}$. With this choice we finally obtain $\bar{\kappa}$ such that, for all $\kappa \geqslant \bar{\kappa}$, we have

$$
\left[f_{i}^{\prime}(x, 0)-\kappa\left(u_{h}^{\kappa}+u_{h}^{0}\right)\right]^{+}(x)=0
$$

for any $x$ in $B_{h}(r) \backslash A_{\delta}^{\kappa}$. Summing up, the above argument yields

$$
\begin{aligned}
\left\|\left[f_{i}^{\prime}(\cdot, 0)-\kappa\left(u_{h}^{\kappa}+u_{h}^{0}\right)\right]^{+}\right\|_{L^{N / 2}\left(B_{h}\right)}^{N / 2} \leqslant & \left\|\left[f_{i}^{\prime}(\cdot, 0)-\kappa\left(u_{h}^{\kappa}+u_{h}^{0}\right)\right]^{+}\right\|_{L^{N / 2}\left(B_{h}(r) \backslash A_{\delta}^{\kappa}\right)}^{N / 2} \\
& +\left\|\left[f_{i}^{\prime}(\cdot, 0)-\kappa\left(u_{h}^{\kappa}+u_{h}^{0}\right)\right]^{+}\right\|_{L^{N / 2}\left(\left(B_{h} \backslash B_{h}(r)\right) \cup A_{\delta}^{\kappa}\right)}^{N / 2} \\
\leqslant & \frac{1}{2}(S(1-\varepsilon))^{N / 2}
\end{aligned}
$$

for $\kappa$ large enough, and proves the Claim.

As a consequence, if $\kappa$ is large enough, we obtain $\left.v_{i}^{\kappa}\right|_{B_{h}} \equiv 0$ for all $h \neq i$. Making use of this in 26) for the choice $h=i$ we get

$$
\int_{B_{i}}\left(\left|\nabla v_{i}^{\kappa}\right|^{2}-f_{i}^{\prime}\left(x, u_{i}^{0}\right)\left|v_{i}^{\kappa}\right|^{2}\right)=\int_{B_{i}}\left(f_{i}\left(x, u_{i}^{\kappa}\right)-f_{i}\left(x, u_{i}^{0}\right)-f_{i}^{\prime}\left(x, u_{i}^{0}\right) v_{i}^{\kappa}\right) v_{i}^{\kappa}
$$

for $\kappa$ large enough. In light of assumption (ND) the left hand side is always greater than $\varepsilon\left\|v_{i}^{\kappa}\right\|_{H_{0}^{1}\left(B_{i}\right)}^{2}$ for some positive $\varepsilon$. On the other hand, Lemma 6.1 ensures that the right hand side is less than $(\varepsilon / 2)\left\|v_{i}^{\kappa}\right\|_{H_{0}^{1}\left(B_{i}\right)}^{2}$ if $\left\|v_{i}^{\kappa}\right\|_{H_{0}^{1}\left(B_{i}\right)}$ is suitably small. Hence we reach a contradiction for $\kappa$ large enough unless $v_{i}^{\kappa} \equiv 0$ for all $i=1, \ldots, k$, that is, $U^{\kappa} \equiv U^{0}$. 
Proof of Lemma 2.3. Let $i$ be fixed and consider the differential inequality

$$
-\Delta \widehat{u}_{i} \geqslant \widehat{f}_{i}\left(x, \widehat{u}_{i}\right) \quad \text { in } B_{i} .
$$

Testing it with $-\widehat{u}_{i}^{-}$and setting $\omega_{i}:=\left\{\widehat{u}_{i}^{-}>0\right\}$ yields

$$
\int_{\omega_{i}}\left|\nabla\left(\widehat{u}_{i}\right)\right|^{2} \leqslant-\int_{\omega_{i}} \frac{\widehat{f_{i}}\left(x, \widehat{u}_{i}\right)}{\widehat{u}_{i}}\left(u_{i}^{-}\right)^{2} \leqslant M \mu\left(\omega_{i}\right)^{2 / N} S^{-1} \int_{\omega_{i}}\left|\nabla\left(\widehat{u}_{i}\right)^{2}\right|,
$$

where $M:=\left\|\widehat{f_{i}}\left(x, \widehat{u}_{i}^{-}\right) / \widehat{u}_{i}\right\|_{L^{\infty}}$ is finite by the a priori $L^{\infty}$-estimate for $u_{i}$ as in 10 and taking into account that $f_{j}^{\prime}(0)$ is finite for all $j$. Now, since $\left\|\widehat{u}_{i}-u_{i}^{0}\right\|_{H_{0}^{1}\left(B_{i}\right)} \leqslant k \delta$, we have

$$
k^{2} \delta^{2} \geqslant \int_{B_{i}}\left|\nabla\left(\widehat{u}_{i}-u_{i}^{0}\right)\right|^{2} \geqslant \operatorname{const}\left(\int_{B_{i}}\left|\widehat{u}_{i}-u_{i}^{0}\right|^{2^{*}}\right)^{2 / 2^{*}} \geqslant \operatorname{const}\left(\int_{\omega_{i}}\left|u_{i}^{0}\right|^{2^{*}}\right)^{2 / 2^{*}} .
$$

By absolute continuity of Lebesgue integral, we can choose $\delta$ sufficiently small to ensure that $\mu\left(\omega_{i}\right)<(S / 2 M)^{N / 2}$. Hence by 27 we find

$$
\int_{\omega_{i}}\left|\nabla\left(\widehat{u}_{i}^{-}\right)\right|^{2} \leqslant \frac{1}{2} \int_{\omega_{i}}\left|\nabla\left(\widehat{u}_{i}^{-}\right)^{2}\right|,
$$

which implies $\widehat{u}_{i} \equiv 0$.

Proof of Theorem 2.2 By Lemma 2.3 we know that $\widehat{u}_{i} \geqslant 0$ in $B_{i}$ for all $i$. Since $u_{j} \geqslant 0$ for all $j$ and the supports are disjoint, $\sum_{j \neq i} u_{j}=\left(\widehat{u}_{i}\right)^{-}=0$, implying $\widehat{u}_{i} \equiv u_{i}$. Hence by coupling the differential inequalities for $u_{i}$ and $\widehat{u}_{i}$ we find that $u_{i}$ is a solution to

$$
-\Delta u_{i}=f_{i}\left(x, u_{i}\right) \quad \text { in } B_{i},
$$

with null boundary conditions. Hence by assumption (ND) we obtain $u_{i} \equiv u_{i}^{0}$.

The following lemma establishes the Fréchet differentiability of the map $F^{0, \kappa}$ defined in Section 5 LEMMA 6.2 For any $r \in\left[\frac{2 N \tilde{q}}{N+2}, \frac{2 N}{N-2}\right]$, the Nemytskiı̌ operator

$$
\begin{aligned}
& F^{0, \kappa}:\left(L^{r}\left(\Omega^{0}\right)\right)^{k} \rightarrow\left(L^{r / \tilde{q}}\left(\Omega^{0}\right)\right)^{k}, \\
& F^{0, \kappa}(U)=f_{i}\left(\cdot,\left[u_{i}+u_{i}^{0}\right]^{+}-u_{i}^{0}\right)-\kappa\left[u_{i}+u_{i}^{0}\right]^{+} \sum_{j \neq i}\left[u_{j}+u_{j}^{0}\right]^{+},
\end{aligned}
$$

is Fréchet differentiable at $U^{0}$ and

$$
\left(F^{0, \kappa}\right)^{\prime}\left(U^{0}\right)[V]=\left(\left(f_{i}^{\prime}\left(\cdot, u_{i}^{0}\right)-2 \kappa \sum_{j \neq i} u_{j}^{0}\right) v_{i}-2 \kappa u_{i}^{0} \sum_{j \neq i} v_{j}\right)_{i=1, \ldots, k} .
$$

Proof. We shall prove that for all $i=1, \ldots, k$,

$$
\begin{aligned}
\| f_{i}\left(\cdot,\left[u_{i}+\right.\right. & \left.\left.u_{i}^{0}\right]^{+}-u_{i}^{0}\right)-f_{i}\left(\cdot, u_{i}^{0}\right)-f_{i}^{\prime}\left(\cdot, u_{i}^{0}\right)\left(u_{i}-u_{i}^{0}\right) \\
& -\kappa\left[\left[u_{i}+u_{i}^{0}\right]^{+} \sum_{j \neq i}\left[u_{j}+u_{j}^{0}\right]^{+}-2\left(\sum_{j \neq i} u_{j}^{0}\right)\left(u_{i}-u_{i}^{0}\right)-2 u_{i}^{0} \sum_{j \neq i}\left(u_{j}-u_{j}^{0}\right)\right] \|_{\left(L^{r / \tilde{q}}\left(\Omega^{0}\right)\right)^{k}} \\
= & o\left(\left\|U-U^{0}\right\|_{\left.\left(L^{r}\left(\Omega^{0}\right)\right)^{k}\right)} \text { as }\left\|U-U^{0}\right\|_{\left(L^{r}\left(\Omega^{0}\right)\right)^{k}} \rightarrow 0 .\right.
\end{aligned}
$$


We have

$$
\frac{\left\|f_{i}\left(\cdot,\left[u_{i}+u_{i}^{0}\right]^{+}-u_{i}^{0}\right)-f_{i}\left(\cdot, u_{i}^{0}\right)-f_{i}^{\prime}\left(\cdot, u_{i}^{0}\right)\left(u_{i}-u_{i}^{0}\right)\right\|_{\left(L^{r / \tilde{q}}\left(\Omega^{0}\right)\right)^{k}}^{r / \tilde{q}}}{\left\|u_{i}-u_{i}^{0}\right\|_{\left(L^{r}\left(\Omega^{0}\right)\right)^{k}}^{r / \tilde{q}}} \leqslant I_{1}+I_{2},
$$

where

$$
\begin{aligned}
& I_{1}=\frac{\int_{\left\{u_{i}+u_{i}^{0}>0\right\}}\left|f_{i}\left(\cdot, u_{i}\right)-f_{i}\left(\cdot, u_{i}^{0}\right)-f_{i}^{\prime}\left(\cdot, u_{i}^{0}\right)\left(u_{i}-u_{i}^{0}\right)\right|^{r / \tilde{q}}}{\left(\int_{\Omega^{0}}\left|u_{i}-u_{i}^{0}\right|^{r}\right)^{1 / \tilde{q}}}, \\
& I_{2}=\frac{\int_{\left\{u_{i}+u_{i}^{0}<0\right\}}\left|2 f_{i}\left(\cdot, u_{i}^{0}\right)+f_{i}^{\prime}\left(\cdot, u_{i}^{0}\right)\left(u_{i}-u_{i}^{0}\right)\right|^{r / \tilde{q}}}{\left(\int_{\left\{u_{i}+u_{i}^{0}<0\right\}}\left|u_{i}-u_{i}^{0}\right|^{r}\right)^{1 / \tilde{q}}} .
\end{aligned}
$$

Mimicking the proof of Lemma 6.1 we can easily show that $I_{1} \rightarrow 0$ as $u_{i} \rightarrow u_{i}^{0}$ in $L^{r}(\Omega)$. Setting $\omega_{i}:=\left\{x \in \Omega: u_{i}(x)+u_{i}^{0}(x)<0\right\}$, we observe that

hence $\left|\omega_{i}\right| \rightarrow 0$ as $u_{i} \rightarrow u_{i}^{0}$ in $L^{r}(\Omega)$.

$$
\int_{\Omega}\left|u_{i}-u_{i}^{0}\right|^{r} \geqslant \int_{\omega_{i}}\left|u_{i}^{0}\right|^{r}
$$

From assumptions $(\mathrm{F} 1),(\mathrm{F} 2)$ we find that $f_{i}(x, s) \leqslant$ const $\left(|s|+|s|^{\tilde{q}}\right)$, hence

$$
\begin{aligned}
\frac{\left(\int_{\omega_{i}}\left|f_{i}\left(\cdot, u_{i}^{0}\right)\right|^{r / \tilde{q}}\right)^{\tilde{q} / r}}{\left(\int_{\omega_{i}}\left|u_{i}-u_{i}^{0}\right|^{r}\right)^{1 / r}} & \leqslant \frac{\left(\int_{\omega_{i}}\left|u_{i}^{0}\right|^{r / \tilde{q}}\right)^{\tilde{q} / r}}{\left(\int_{\omega_{i}}\left|u_{i}^{0}\right|^{r}\right)^{1 / r}}+\frac{\left(\int_{\omega_{i}}\left|u_{i}^{0}\right|^{r}\right)^{\tilde{q} / r}}{\left(\int_{\omega_{i}}\left|u_{i}^{0}\right|^{r}\right)^{1 / r}} \\
& \leqslant\left|\omega_{i}\right|^{(\tilde{q}-1) / r}+\left(\int_{\omega_{i}}\left|u_{i}^{0}\right|^{r}\right)^{(\tilde{q}-1) / r}=o(1)
\end{aligned}
$$

as $u_{i} \rightarrow u_{i}^{0}$ in $L^{r}(\Omega)$. Moreover,

$$
\frac{\left(\int_{\omega_{i}}\left|f_{i}^{\prime}\left(\cdot, u_{i}^{0}\right)\left(u_{i}-u_{i}^{0}\right)\right|^{r / \tilde{q}}\right)^{\tilde{q} / r}}{\left(\int_{\omega_{i}}\left|u_{i}-u_{i}^{0}\right|^{r}\right)^{1 / r}} \leqslant \frac{\left\|u-u_{i}^{0}\right\|_{L^{r}\left(\omega_{i}\right)}\left\|f_{i}^{\prime}\left(\cdot, u_{i}^{0}\right)\right\|_{L^{r /(\tilde{q}-1)}\left(\omega_{i}\right)}}{\left\|u-u_{i}^{0}\right\|_{L^{r}\left(\omega_{i}\right)}}=o(1)
$$

as $u_{i} \rightarrow u_{i}^{0}$ in $L^{r}(\Omega)$. From $\sqrt{28}$ and 29 it follows that $I_{2}=o(1)$ as $u_{i} \rightarrow u_{i}^{0}$ in $L^{r}(\Omega)$. Hence

$$
\left\|f_{i}\left(\cdot,\left[u_{i}+u_{i}^{0}\right]^{+}-u_{i}^{0}\right)-f_{i}\left(\cdot, u_{i}^{0}\right)-f_{i}^{\prime}\left(\cdot, u_{i}^{0}\right)\left(u_{i}-u_{i}^{0}\right)\right\|_{\left(L^{r / \tilde{q}}\left(\Omega^{0}\right)\right)^{k}}=o\left(\left\|u_{i}-u_{i}^{0}\right\|_{\left(L^{r / \tilde{q}}\left(\Omega^{0}\right)\right)^{k}}\right.
$$
as $u_{i} \rightarrow u_{i}^{0}$ in $L^{r}(\Omega)$. On the other hand,

$$
\begin{aligned}
& \left\|\left[u_{i}+u_{i}^{0}\right]^{+} \sum_{j \neq i}\left[u_{j}+u_{j}^{0}\right]^{+}-2\left(\sum_{j \neq i} u_{j}^{0}\right)\left(u_{i}-u_{i}^{0}\right)-2 u_{i}^{0} \sum_{j \neq i}\left(u_{j}-u_{j}^{0}\right)\right\|_{\left(L^{r / \tilde{q}}\left(\Omega^{0}\right)\right)^{k}} \\
& \leqslant \sum_{j \neq i}\left(\int_{\Omega^{0} \backslash\left(\omega_{i} \cup \omega_{j}\right)}\left|u_{i}-u_{i}^{0}\right|^{r / \tilde{q}}\left|u_{j}-u_{j}^{0}\right|^{r / \tilde{q}}+\int_{\omega_{i} \cup \omega_{j}}\left|2 u_{j}^{0}\left(u_{i}-u_{i}^{0}\right)+2 u_{i}^{0}\left(u_{j}-u_{j}^{0}\right)\right|^{r / \tilde{q}}\right)^{\tilde{q} / r} \\
& \leqslant \mathrm{const} \sum_{j \neq i}\left(\left\|u_{i}-u_{i}^{0}\right\|_{L^{r}(\Omega)}^{r / \tilde{q}}\left\|u_{j}-u_{j}^{0}\right\|_{L^{r /(\tilde{q}-1)}(\Omega)}^{r / \tilde{q}}\right. \\
& \left.\quad+\left\|u_{i}-u_{i}^{0}\right\|_{L^{r}(\Omega)}^{r / \tilde{q}}\left\|u_{j}^{0}\right\|_{L^{r /(\tilde{q}-1)}\left(\omega_{i} \cup \omega_{j}\right)}^{r / \tilde{r}}+\left\|u_{j}-u_{j}^{0}\right\|_{L^{r}(\Omega)}^{r / \tilde{q}}\left\|u_{i}^{0}\right\|_{L^{r /(\tilde{q}-1)}\left(\omega_{i} \cup \omega_{j}\right)}^{r / \tilde{q}}\right)^{\tilde{q} / r} \\
& =o(1) \quad \text { as }\left\|U-U^{0}\right\|_{\left(L^{r}\left(\Omega^{0}\right)\right)^{k}} \rightarrow 0 .
\end{aligned}
$$

The proof is thereby complete. 
Since we are actually working with the truncation $\tilde{f}_{i}$ instead of $f_{i}$, and $\tilde{f}_{i}$ is not $C^{1}$ with respect to the second variable, it is worth noticing that this does not create any problem when linearizing the operator at $U^{0}$ and the linearization of the truncated operator is still given by 20]. As the proof is very similar to the proof of Lemma 6.2, we omit it.

\section{Acknowledgments}

The authors wish to express their gratitude to Prof. Susanna Terracini for interesting comments and discussions. They are also indebted to the anonymous referees for their helpful remarks.

This research was partially supported by the Italian MIUR Research Project Metodi Variazionali ed Equazioni Differenziali Nonlineari.

\section{REFERENCES}

1. Conti, M., Terracini, S., \& Verzini, G. Nehari's problem and competing species systems. Ann. Inst. H. Poincaré 19 (2002), 871-888. Zbl 1090.35076 MR 1939088

2. Conti, M., Terracini, S., \& Verzini, G. An optimal partition problem related to nonlinear eigenvalues. J. Funct. Anal. 198 (2003), 160-196. Zbl 1091.35051 MR 1962357

3. Conti, M., Terracini, S., \& Verzini, G. A variational problem for the spatial segregation of reaction-diffusion systems. Indiana Univ. Math. J. 54 (2005), 779-815. Zbl pre02204353 MR 2151234

4. Conti, M., Terracini, S., \& Verzini, G. On a class of optimal partition problems related to the Fučík spectrum and to the monotonicity formulae. Calc. Var. Partial Differential Equations 22 (2005), 45-72. Zbl pre02135754 MR 2105968

5. Conti, M., Terracini, S., \& Verzini, G. Asymptotic estimates for the spatial segregation of competitive systems. Adv. Math. 195 (2005), 524-560. Zbl 1126.35016 MR 2146353

6. Conti, M., Terracini, S., \& Verzini, G. A regularity theory for optimal partition problems. SPT 2004 - Symmetry and Perturbation Theory, World Sci., Hackensack, NJ (2005), 91-98. MR 2331210

7. Conti, M., Terracini, S., \& Verzini, G. Uniqueness and least energy property for solutions to strongly competing systems. Interfaces Free Bound. 8 (2006), 437-446. Zbl 1103.92041 MR 2283921

8. DANCER, E. N. The effect of domain shape on the number of positive solutions of certain nonlinear equations. J. Differential Equations 74 (1988), 120-156. Z Zbl 0662.34025 MR 0949628

9. DANCER, E. N., \& DU, Y. H. Competing species equations with diffusion, large interactions, and jumping nonlinearities. J. Differential Equations 114 (1994), 434-475. Zbl 0815.35024 MR 1303035

10. DANCER, E. N., \& DU, Y. H. Positive solutions for a three-species competition system with diffusion. I. General existence results. Nonlinear Anal. 24 (1995), 337-357. Zbl 0824.35033 MR 1312772

11. Dancer, E. N., \& DU, Y. H. Positive solutions for a three-species competition system with diffusion. II. The case of equal birth rates. Nonlinear Anal. 24 (1995), 359-373. Zbl 0824.35034 MR 1312773

12. DANCER, E. N., \& GUO, Z. M. Uniqueness and stability for solutions of competing species equations with large interactions. Comm. Appl. Nonlinear Anal. 1 (1994), 19-45. Zbl 0935.35074 MR 1280113

13. Dancer, E. N., Hilhorst, D., Mimura, M., \& Peletier, L. A. Spatial segregation limit of a competition-diffusion system. Eur. J. Appl. Math. 10 (1999), 97-115. Zbl 0982.92031 MR 1687440

14. EI, S. I., FANG, Q., \& MimURA, M. Effect of domain shape on coexistence problems in a competitiondiffusion system. J. Math. Biol. 29 (1991), 219-237. Zbl 0737.92024 MR 1089783

15. Eilbeck, J. C., Furter, J. E., \& López-GómeZ, J. Coexistence in the competition model with diffusion. J. Differential Equations 107 (1994), 96-139. Zbl 0833.92010 MR 1260851

16. Fonseca, I., \& Gangbo, W. Degree Theory in Analysis and Applications. Oxford Lecture Ser. Math. Appl. 2, Oxford Univ. Press, New York (1995). Zbl 0852.47030 MR 1373430 
17. Gilbarg, D., \& Trudinger, N. Elliptic Partial Differential Equations of Second Order. Springer, Berlin (1977). Zbl 0361.35003 MR 0473443

18. Gui, C., \& LOU, Y. Uniqueness and nonuniqueness of coexistence states in the Lotka-Volterra competition model. Comm. Pure Appl. Math. 47 (1994), 1571-1594. Zbl 0829.92015 MR 1303221

19. Kishimoto, K., \& Weinberger, H. F. The spatial homogeneity of stable equilibria of some reactiondiffusion system on convex domains. J. Differential Equations 48 (1985), 15-21. Zbl 0599.35080 MR 0791838

20. Korman, P., \& Leung, A. On the existence and uniqueness of positive steady states in Lotka-Volterra ecological models with diffusion. Appl. Anal. 26 (1987), 145-160. Zbl 0639.35026 MR 0921723

21. LAZER, A. C., \& MCKenna, P. J. On steady state solutions of a system of reaction-diffusion equations from biology. Nonlinear Anal. 6 (1982), 523-530. Zbl 0488.35039 MR 0664014

22. Lou, Y., Martínez, S., \& Ni, W.-M. On $3 \times 3$ Lotka-Volterra competition systems with crossdiffusion. Discrete Contin. Dynam. Systems 6 (2000), 175-190. Zbl 1008.92035 MR 1739922

23. Lou, Y., \& NI, W.-M. Diffusion, self-diffusion and cross-diffusion. J. Differential Equations 131 (1996), 79-131. Zbl 0867.35032 MR 1415047

24. Matano, H., \& Mimura, M. Pattern formation in competition-diffusion systems in nonconvex domains. Publ. Res. Inst. Math. Sci. 19 (1983), 1049-1079. Zbl 0548.35063 MR 0723460

25. Shigesada, N., KaWASAKi, K., \& Teramoto, E. The effects of interference competition on stability, structure and invasion of a multispecies system. J. Math. Biol. 21 (1984), 97-113. Zbl 0568.92017 MR 0782629

26. SweErs, G. A sign-changing global minimizer on a convex domain. Progress in Partial Differential Equations: Elliptic and Parabolic Problems (Pont-à-Mousson, 1991), Pitman Res. Notes Math. Ser. 266, Longman Sci. Tech., Harlow (1992), 251-258. Zbl 0789.35066 MR 1194233 